\title{
Studying Individual Events in Biology
}

\section{Stefan Wennmalm and Sanford M. Simon}

Laboratory of Cellular Biophysics, The Rockefeller University, New York, New York 10021; email: simon@rockefeller.edu

Annu. Rev. Biochem. 2007. 76:419-46

First published online as a Review in Advance on March 22, 2007

The Annual Review of Biochemistry is online at biochem.annualreviews.org

This article's doi:

10.1146/annurev.biochem.76.062305.094225

Copyright (c) 2007 by Annual Reviews.

All rights reserved

0066-4154/07/0707-0419\$20.00

\section{Key Words}

endocytosis, exocytosis, imaging, quantum dots, single molecule

\begin{abstract}
Studying the properties of individual events and molecules offers a host of advantages over taking only macroscopic measurements of populations. Here we review such advantages, as well as some pitfalls, focusing on examples from biological imaging. Examples include single proteins, their interactions in cells, organelles, and their interactions both with each other and with parts of the cell. Additionally, we discuss constraints that limit the study of single events, along with the criteria that must be fulfilled to determine whether single molecules or events are being detected.
\end{abstract}




\section{Contents}

INTRODUCTION............. 420

HISTORICAL BACKGROUND ... 423

Imaging of Organelles Moving

Along Filaments............. 424

Single Motor Protein Molecules .. 424

Single Molecules and Fluorophores

in Solution................ 424

Single Particles on Surfaces of

Cells...................... 425

Single Molecules in Cells ........ 425

ADVANTAGES OF STUDYING

SINGLE EVENTS ............ 426

Detecting Biologically Relevant

Subpopulations............. 426

The Possibility of Observing

Molecular Events without

Synchronization............ 427

PITFALLS IN SINGLE-EVENT

DETECTION ................ 431

Are Labeled Molecules

Functional? .............. 432

Criteria for Identifying Single

Molecules................ 432

DOES THE SIGNAL REPORTED

BY OUR ASSAY REPRESENT

THE SINGLE EVENT OF

INTEREST? ............... 436

Protein Translocation Across

Membranes/Export.......... 437

Membrane Exocytosis/

Endocytosis.............. 437

WHEN IS A RARE ANECDOTAL

EVENT SIGNIFICANT? ....... 439

CONCLUSION AND

OUTLOOK

Endocytosis: A process by which cells internalize molecules from the outside and the cell surface through invagination of membrane for Einstein's description of diffusion as the consequence of single molecules taking random walks (2).

Advances in digital electronics have increased the number of different assays that can detect single events and even single molecules. Most of these technologies rely on the modalities of electrophysiology or imaging. Patchclamp electrophysiology can measure single ion channels as they open and close; wholecell-clamp electrophysiology can monitor the release of a single quantum of a transmitter in synapses; and amperometry electrophysiology can follow the release of the contents of single secretory vesicles. Meanwhile, fluorescence-based imaging can study processes such as the exo- and endocytosis of single vesicles, the dynamics of conformational changes of single $\mathrm{H}^{+}$-ATP-ases, and the movement of single molecular motors. Atomic-force-microscopy imaging yields insights into the shape and conformations of individual macromolecules.

There are several advantages to studying single events and molecules:

- A particular population may exist in two separate states. A measurement of its macroscopic behavior reports only the average, whereas none of its members actually performs the average behavior.

- Many biological states are transient, which may not be represented in macroscopic measurements of the average population. The macroscopic measurements may reflect the dominant population in the system, but not the biologically active individuals.

- Relying on macroscopic measurements and population synchronization makes it difficult to detect the temporal order of a collection of transient events. Synchronization becomes unnecessary when using measurements of single events.

- The results of macroscopic measurements can often be interpreted in multiple ways, where different microscopic 
events could account for the macroscopic observations. Studying single events and molecules allows us to test and disprove the possibilities. For example, when single vesicles in the neuromuscular junction release acetylcholine, it binds to channels that initiate a postsynaptic response that rises quickly, then decays exponentially. Macroscopic measurements led to two possible explanations. First, many channels may open simultaneously, followed by their amplitude dropping exponentially. Second, channels may open to constant amplitude and remain open for variable times whose duration is distributed exponentially (3). The observation of the first single acetylcholine channels immediately demonstrated that the latter model is correct (4).

To illustrate these advantages of studying individual events, we use two examples from the study of exocytosis, the fusion of a vesicle to the plasma membrane of a cell. Studies on the fusion of liposomes to planar lipid bilayers had implicated osmotic forces in membrane fusion (5). In 1987, one critical question regarding the mechanism of exocytosis was whether secretory vesicles swell prior to fusing to the membrane, providing a driving force for membrane fusion, or if they swell only after fusion to the membrane as a consequence of water rushing in through the fusion pore and hydrating the contents of the vesicle.

Using the patch-clamp technique, one can determine the moment a single vesicle fuses to the plasma membrane by quantifying the capacitance of the cell's plasma membrane. The fusion of each vesicle leads to an increase in the surface area of the cell and thus an increase in the capacitance of the plasma membrane. The swelling of the vesicle can be monitored using video microscopy.

In one approach to answer the question of whether swelling occurs before or after fusion, researchers stimulated membrane fu- sion, measured the average time until vesicles fused by using the increased capacitance, and then compared this number with the average time it took the cell to swell based on the video measurement. The average time between stimulation of secretion and membrane fusion was $3 \pm 2 \mathrm{~min}$. The average time between the stimulation of secretion and vesicle swelling was also $3 \pm 2 \mathrm{~min}$. Given the large variability in the time of fusion and the time of swelling, the two results were indistinguishable (6).

In an alternative approach, researchers monitored individual fusion events and quantified the time difference between when each fusion initiated (based on capacitance) and when swelling started (as monitored by video microscopy). When this approach was applied to the study of exocytosis in mast cells from the beige mouse, swelling always occurred after membrane fusion $(6,7)$. The average time between fusion and swelling was just over $100 \mathrm{~ms}$ (an example is shown in Figure 1) significantly smaller than the variation in the measurements of the time to fusion or of the time to swelling. This illustrates why this value could not be determined by calculating the difference between the average time to swelling and the average time to fusion. Investigators were able to resolve the differences between these hypotheses by studying single exocytic events.

A second example of the benefit of examining single events to study exocytosis comes from studies of calcium's role in the process of transmitter release at the presynaptic terminal. Transient changes of intracellular calcium may play a critical role in many different signaling pathways. Since the mid-1800s, it has been known that calcium is essential in the extracellular bath to maintain neuromuscular transmission (8). Because calcium must be present at the precise moment at which an action potential passes through the terminal, researchers realized it was likely needed for a specific signaling function rather than just general viability (9). Using aequorin, calcium could be imaged entering the terminal in a fashion that qualitatively predicted the time
Exocytosis: the process by which cells secrete molecules, or add new molecules to their surface, by fusing a membrane surrounding a packet of molecules inside the cell to the membrane at the cell surface

Secretion: the process by which cells release molecules 
a
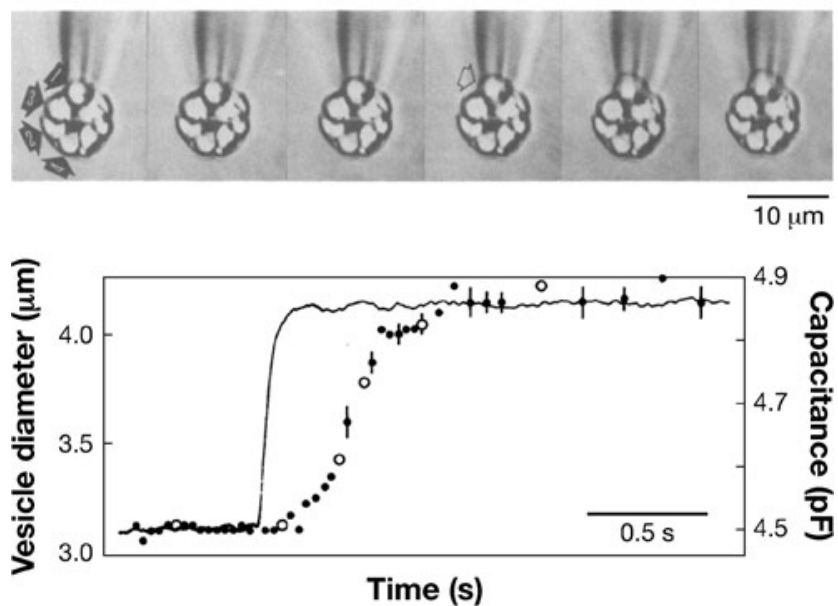

b

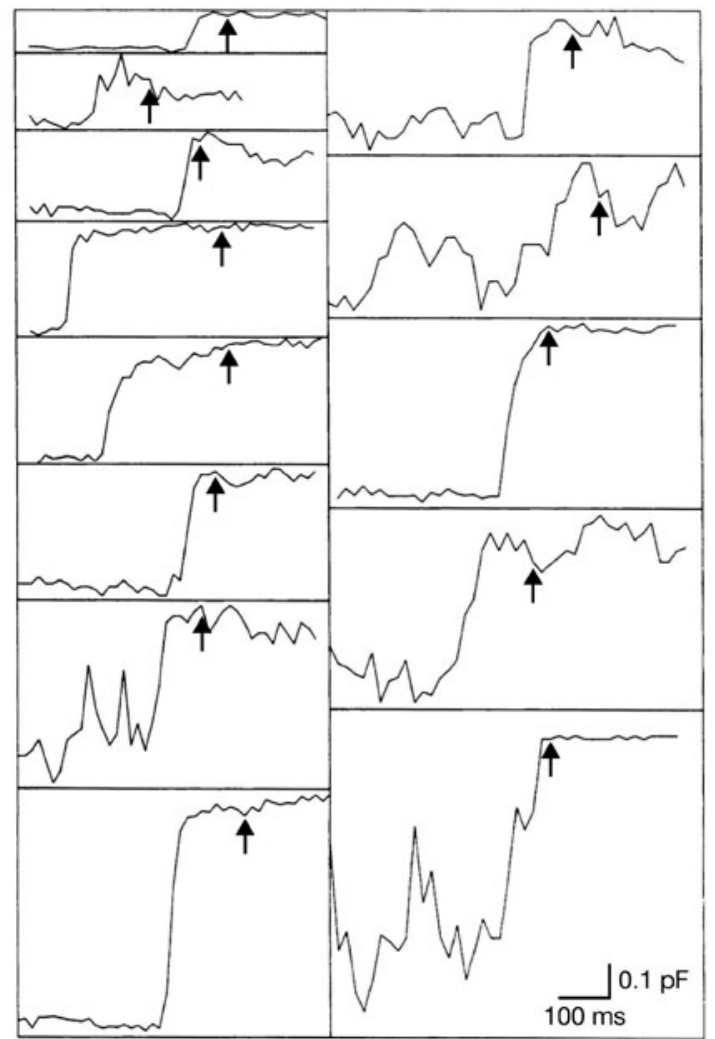

Figure 1

Membrane fusion (solid line), as assayed by membrane capacitance, always precedes vesicles swelling (circles in panel $a$, arrows in panel $b$ ). Panel $a$ reprinted from Breckenridge \& Almers, PNAS 84:1585-89 (7), and panel $b$ reprinted from Zimmerberg et al., PNAS 84:1585-89 (6), with permission. course and magnitude of transmitter release by exocytosis (10).

Surprisingly, the magnitude of the calcium entering the terminal did not quantitatively account for the time course and magnitude of transmitter release (11). When the voltage across the membrane of the presynaptic terminal of a nerve was raised from its resting potential, the calcium channels opened, allowing calcium to enter the cell. With larger voltage displacement from rest, more channels opened (Figure $2 \boldsymbol{a}$, red line). However, making the inside of the nerve become more positive also decreases the driving force for positively charged calcium to enter and thus less calcium enters per channel (Figure $2 a$, blue line). Once the membrane potential was depolarized beyond zero, the total calcium entry decreased even though more calcium channels opened (Figure $\mathbf{2} \boldsymbol{a}$, gold line). The plot of total calcium current as a function of voltage has a bell shape. Thus, macroscopically, the total calcium current has the same value twice during the process. However, the amount of transmitter release that occurred at more positive membrane potentials was considerably greater than the release that occurred at more negative potentials. This suggests a significant effect of membrane potential on transmitter release.

The conclusion that a disparity exists between the amount of calcium entering and the amount of transmitter release by exocytosis was based on this observation: Two different membrane potentials that yield the same macroscopic calcium current (e.g., $+20 \mathrm{mV}$ and $-20 \mathrm{mV}$ ) (Figure $2 \boldsymbol{a}$ ) do not yield the same amount of transmitter release. However, when the calcium currents are considered on a microscopic level, the difference between them is significant (Figure $\mathbf{2} \boldsymbol{b}, \boldsymbol{c}$ ). At $-20 \mathrm{mV}$, there are a few channels open, with a large flux through each channel, whereas at $+20 \mathrm{mV}$, the same total current enters through many more channels. The distance over which the calcium concentration increases is comparable with the size of a secretory vesicle. In the extreme case of only 
one channel being open, with a large influx through that channel, only one vesicle can be released. When the same amount of calcium enters through many channels, many more vesicles can be released. This model, which requires examining calcium entry at the individual channel level, quantitatively accounts for the magnitude and kinetics of transmitter release (12), and the principle of examining localized changes of calcium activity is now widely accepted.

This review focuses on the types of studies of individual events in biology that have become possible with sensitive imaging techniques since the late 1980s and early 1990s. Because of this we limit the background description to the work that directly preceded those studies. Regarding the detection of single molecules, several review articles exist (1315). We focus more generally on the imaging of single events, the specific advantages offered by the study of single events, and also on some of the potential liabilities and caveats that accompany such studies. In addition we discuss criteria that can be used to determine if single events and single molecules are being detected.

\section{HISTORICAL BACKGROUND}

One field that has benefited significantly from sensitive fluorescence imaging techniques is the study of molecular motors. Because of this we start the historical overview with the

\section{Figure 2}

(a) With the calcium currents quantified and the fraction opened (red line), the flux through each channel (blue line) and the total macroscopic current (gold line) are plotted as a function of voltage. The magnitude of the total calcium current entering the neuron is the same at $-20 \mathrm{mV}$ and $+20 \mathrm{mV}$. However, the nature of the calcium entry, on a microscopic level, is quite different. (b) At $-20 \mathrm{mV}$ there are fewer channels open with much more calcium entering. (c) At $-20 \mathrm{mV}$ many more calcium channels are open, with less calcium entering through each channel. Figure adapted from Simon \& Llinás (12). imaging of single events of organelle transport along filaments in the 1980s, which involved the molecular motors myosin and kinesin.

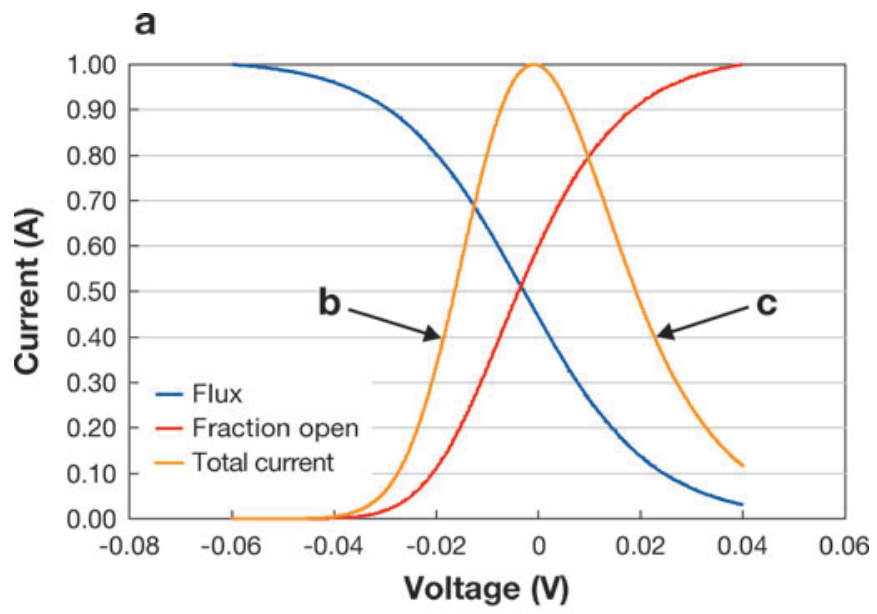

b

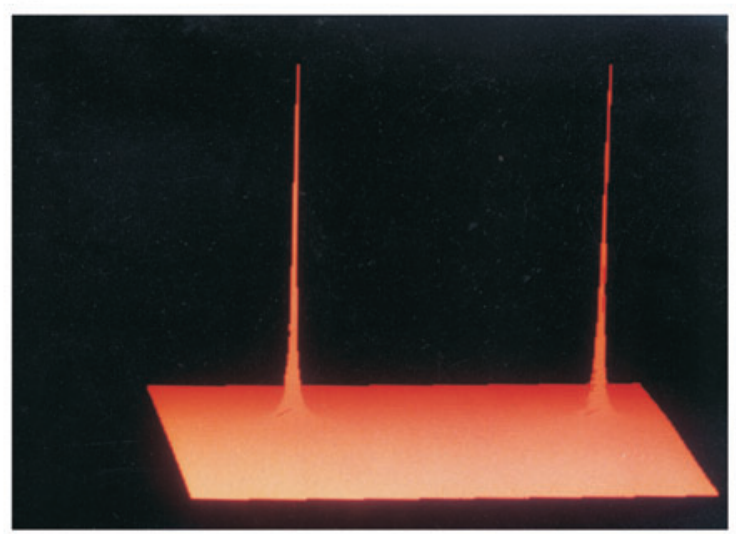

C

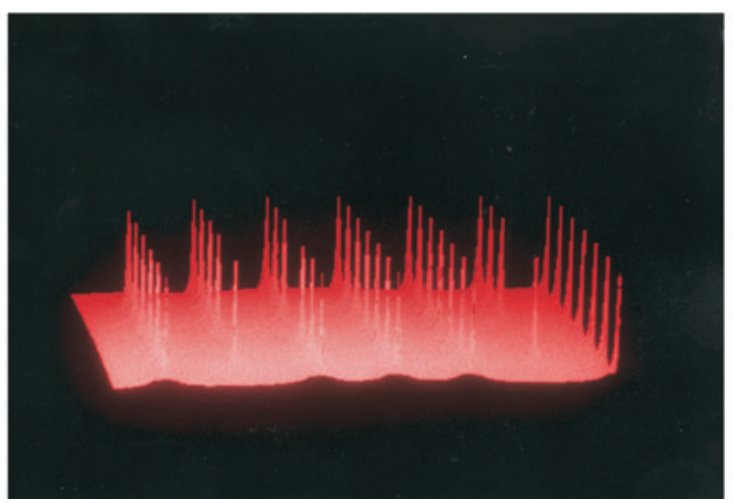


DIC: differential interference contrast

\section{Imaging of Organelles Moving Along} Filaments

In 1982, before the motor kinesin had even been identified, "submicroscopic particles," presumably vesicles $30-50 \mathrm{~nm}$ in diameter, were detected moving parallel to linear elements in squid giant axons (16). In 1985, single organelles were imaged moving along filaments from squid giant axons, single beads moving along filaments, and filaments moving along glass by using differential interference contrast (DIC) microscopy $(17,18)$. Movement only occurred in the presence of axoplasmic supernatant and ATP. With subsequent electron microscopy, these researchers identified the filaments along which organelle movement had been observed as microtubules (17). A detailed study of the transport of organelles and vesicles along microtubules suggested models for microtubule function (19). The protein generating these movements was identified and given the name kinesin (20). Microtubules were not the only cytoskeletal filaments implicated in directed movement. In the algae Nitella, actin and myosin were demonstrated to drive cytoplasmic streaming (21). Also in Nitella, single myosin-coupled fluorescent beads were imaged moving along actin filaments. The movement was shown to be ATP dependent and was blocked by the inactivation of the myosin heads (22).

\section{Single Motor Protein Molecules}

The imaging of single organelles allowed the study of two molecular motors: kinesin for movement along microtubules and myosin for movement along actin filaments. The next step of elucidating the mechanism by which these motors work required the imaging of single biomolecules. Fluorescent single-actin filaments could be moved by myosin in the presence of ATP. Researchers observed that the actin filaments bended and the motion increased in frequency and amplitude in the presence of myosin fragments and ATP (23), and the filaments moved on a myosin-coated glass surface in the direction of their long axis in an ATP-dependent manner (24). In parallel, investigators made advances with the microtubular motors, including the characterization of discrete step sizes on the nanometer scale. With DIC microscopy, kinesin-coated beads made $\sim 4-n m$ jumps, which were interpreted as the step size for the motor (25). When investigators imaged microtubules being moved by kinesin molecules attached to surfaces, the kinesin maintained its grip $\sim 5 \mu \mathrm{m}$ before the microtubules detached. These measurements also led to a rough estimate of the step size $(15-30 \mathrm{~nm})(26)$. Using optical tweezers, researchers observed that beads covered with kinesin walked $1.4 \mu \mathrm{m}$ along microtubules before leaving the microtubule, corresponding to 140 steps, assuming a $~ 10$-nm step size (27). The use of an optical trapping interferometer subsequently refined the step size for kinesin to $8 \mathrm{~nm}$ (28). This step size has been confirmed (29) and is generally accepted.

These investigations approached the single-molecule level and revealed new results on the mechanism of molecular motors. However, the field of single-molecule detection had to mature for the mechanism of myosin and kinesin to be fully understood (see Molecular Motors, below).

\section{Single Molecules and Fluorophores in Solution}

One of the earliest fluorescence measurements of a single molecule used 80 fluoresceins on a single $\gamma$-globulin molecule (30). The advance to the next level of detection, a single fluorophore, had to await the early 1990s, when techniques for detecting single molecules emanated almost simultaneously: detection in solids using absorption (31) or fluorescence (32) and on surfaces using nearfield scanning optical microscopy (33), as well as excitation with far-field focused laser light. Investigators performed the first observations using far-field focused laser light on flowing or diffusing molecules in solution. Single 
protein molecules of $\beta$-phycoerythrin, a protein containing 34 chromophores, were detected using far-field fluorescence spectroscopy in flowing sample streams (34). With one of the aims being rapid DNA sequencing by the detection of single fluorophore-labeled nucleotide bases, progress was focused on enhanced sensitivity. Shera et al. (35) detected single fluorophores of rhodamine 6G in solution in 1990 (35), and other groups soon confirmed these results with continuously improved signal-to-background ratios $(36,37)$. Researchers demonstrated fluctuations between distinct states in a single molecule by the measurement of the fluorescence lifetime of dye-tagged oligonucleotides (38). Detection and imaging of conformational changes within molecules, and imaging of interactions between single molecules (39), have since then become the main focus in single-molecule studies. Since these first observations, single molecules in solution are now more often observed when being immobilized to surfaces $(40,41)$.

\section{Single Particles on Surfaces of Cells}

After the demonstration of single-molecule detection in vitro, a natural step was to extend such measurements to cells. On cell surfaces, measurements of diffusion had been performed earlier using fluorescence techniques, albeit not on single molecules or even single particles. Biological membranes were then, according to the fluid mosaic model, described as two-dimensional fluids in which integral membrane proteins were free to move. By using fluorescence recovery after photobleaching (FRAP) (42-45), investigators showed that the diffusion of most proteins in cell membranes is restricted orders of magnitude below their theoretical values, suggesting constraints to protein diffusion in membranes (46). Although FRAP is still a commonly used technique for observing membrane diffusion, single-particle tracking and single-molecule detection have helped researchers discover inhomogeneities in cell membranes. Using single 40-nm particles of colloidal gold coupled to membrane proteins, investigators observed that diffusion was largely confined to microdomains $1-2 \mu \mathrm{m}$ in size. Furthermore, an ATP-dependent directed motion was inhibited by cytochalasin, suggesting the involvement of actin (47). Methods were developed for tracking individual fluorescent diI-LDL receptors on cell surfaces and for the estimation of the number of receptors in receptor clusters (48). Single virus particles were tracked inside cells during the 1990s (49, 50); however, the tracking of single molecules on cell surfaces and inside cells had still not been realized at that time.

\section{Single Molecules in Cells}

Even though single molecules could be detected by fluorescence in 1989 and 1990, it took 10 years until single molecules were detected on the surface and inside living cells. Even in 2006, the majority of single-molecule-detection experiments are performed in vitro, owing to the difficulties of performing in vivo measurements (see below). In the mid-1990s, investigators studied the movement of single fluorescently labeled phospholipids in artificial phospholipid membranes $(51,52)$ and used fluorescence correlation spectroscopy to detect fluorescence bursts of single lipid analogs on the surface (53) and inside living cells (54). Starting in 2000, a number of articles marked the first imaging of single molecules in cells. On the surface of cells, lipid probes with saturated but not unsaturated acyl chains associated with domains with confined diffusion, suggesting that these domains were the so-called lipid rafts (55). With total internal reflection fluorescence microscopy, investigators imaged epidermal growth factor receptors on the surface of living cells and showed that dimerization of the receptor occurred prior to the binding of the second epidermal growth factor molecule (56). Inside cells, lampbased epi-microscopy was used to image single R-phycoerythrin molecules in the
FRAP: fluorescence recovery after photobleaching

Total internal reflection: no energy crosses an interface when light hits above a critical angle. However, a standing field is created on the other side that decreases exponentially at fractions of the wavelength of light 
cytoplasm and in the nucleus of living cells (57).

The entry of single adenoviruses into livFRET: fluorescence resonance energy transfer ing cells revealed both diffusive and directed motion of viruses in the cytoplasm and in the nucleus (58). Single influenza virus particles have also been tracked in live cells (59), and analysis of green fluorescent protein-labeled HIV-1 particles showed that HIV uses cytoplasmic dynein to migrate along microtubules toward the nucleus (60). Moreover, the mechanism governing the transport of single molecules in and out of the cell nucleus through nuclear pore complexes has also been analyzed in live cells (61-63).

Although the imaging of single fluorophores is feasible in cells, there are a number of limitations to its application. We cover these limitations in depth below, but two of the critical issues for following fluorophores in cells are the blinking behavior of the fluorophores and the limited time that fluorophores stay in a particular focal plane. If a moving fluorophore cannot be followed consistently from frame to frame, it can be problematic to assume that a fluorophore observed in one frame is the same as a fluorophore that reappears a few frames later.

\section{ADVANTAGES OF STUDYING SINGLE EVENTS}

Individual events in biology could already be observed before the arrival of single-molecule detection. For example, the rise and fall of an action potential are individual events, which could be observed without molecular-scale imaging techniques. However, with the possibility of detecting single molecules and similarly sized objects, the kinds of events and processes being studied by direct imaging have increased substantially. Single-molecule detection shifts the emphasis from studying the product or outcome of systems, such as the outcome of a mutation in a certain gene, to the direct imaging of molecular interactions. The direct imaging of the interaction between units on the molecular level allows strong tests of the function of those units. We discuss two additional major advantages of studying single events below: the possibility of detecting subpopulations and the possibility of studying interactions between molecular units without synchronization.

\section{Detecting Biologically Relevant Subpopulations}

Although ensemble measurements give an estimate of the average property, the detection of single molecular units allows for the entire spectrum of properties or behaviors to be extracted. Thus the existence of subpopulations can be revealed. There are several reasons why the presence of subpopulations would be of biological significance. First, a particular population may exist in two separate states. A measurement of the macroscopic behavior of the population would reveal only the average, and none of the members of the population would demonstrate that average behavior. Second, even if the average does represent the dominant state, often in biology the transient states are significant, which may not be represented in the macroscopic measurements of the average population.

Researchers have shown that single complexes of reverse transcriptase/nucleic acids diffusing freely in solution have more than one functional state. Measurements of fluorescence lifetime, anisotropy, fluorescence emission spectra, and fluorescence resonance energy transfer (FRET) identified structurally distinct forms of complexes, some of which incorporated nucleotides and some of which did not (64).

Diffusion of proteins in a cell. One example of how the macroscopic measurement may not represent any individual subpopulation is from the measurement of the macroscopic diffusion constant of proteins in a cell. One frequently used method for measuring the diffusion constant is $\operatorname{FRAP}(42,44,45,65)$. In this method, a molecule is labeled with a fluorophore, a defined spatial region of the 
cell is photobleached, and the recovery after photobleaching is quantified. The rate of recovery is used to calibrate a diffusion constant. An alternative approach is to track single molecules. From their walk, a diffusion constant can be calibrated. When this approach was applied to phycoerthyrin molecules in cells, there were a number of surprising observations (57). First, the diffusion constant determined from single molecules in cells did not match in vitro measurements of phycoerthyrin in an osmotically matched glycerol solution. Second, the researchers measured a broad non-Gaussian spectrum of diffusion constants from single molecules. They calculated the diffusion constants in two different ways with consistent results: The diffusion constant was not only quite variable between different individuals of the same protein, but it would also change even while following a single protein (57). The results were consistent with proteins continuously engaging and disengaging with various cellular components, which would yield broad spectra of diffusion constants. This suggests that the macroscopic measurements obtained from FRAP gave apparent, not actual, diffusion constants, which were the lumped sum of many proteins in different states (57). The results were confirmed with measurements of protein molecules labeled with four to five fluorophores in the cell nucleus, which also revealed a broad distribution of diffusion constants (66).

Conformational fluctuations in proteins and DNA. Before single-molecule detection, it was difficult to address the relation between the function of larger molecules, such as proteins, and their inherent conformational dynamics. For example, an enzyme's turnover rate may change as the enzyme changes from one conformation to another. If the enzyme has to walk through many conformational substates before a large change in its catalytic activity takes place, a memory effect is observed, so the times required for adjacent catalytic turnovers are not independent. That a protein may exist in different conforma- tional states with different activities had been previously proposed $(67,68)$. With singlemolecule detection, new possibilities for investigating these effects became available because dynamic heterogeneities could now be discriminated from static ones. Macroscopic measurements of a tetramethylrhodamine molecule bound to an 18 mer oligonucleotide showed a biexponential decay of the fluorescence lifetime. In contrast, measurements of single molecules revealed that each molecule displayed either a single exponential decay or a combination of the two lifetimes observed in the ensemble measurement, suggesting fluctuations between different fluorescent states (38). Investigators further analyzed these fluctuations using fluorescence correlation spectroscopy and observed a stretched exponential relaxation behavior, indicating a distribution of relaxation rates $(38,69-71)$. Numerous single-molecule papers are now reporting details on conformational dynamics and protein function. Researchers have studied proteins by measuring the fluorescence from the active site of the enzyme cholesterol oxidase during catalytic turnovers (72), by measuring fluctuations in the fluorescence lifetimes of a flavin when bound to flavin reductase (40), by measuring the enzymatic degradation of single double-stranded DNA molecules to single-stranded DNA by $\lambda$-exonuclease (73), by monitoring the fluorescent products generated by immobilized enzyme molecules (74-77), and by measuring FRET between parts of single hairpin ribozymes, correlating structure to enzymatic function (78).

\section{The Possibility of Observing Molecular Events without Synchronization}

Before biological interactions could be imaged at the molecular level, investigators used different tricks to synchronize processes to observe them. For example, to study the steps in the cell cycle, the cells can be transiently arrested in one phase by disrupting the cytoskeleton or starving the cells of growth 
factors. Upon restoring the cells to a normal growth medium, the cells are relatively in sync (79). For a brief period, changes in the cell cycle could be followed in sequential order. Synchronization could also be accomplished with conditional temperature-sensitive mutants. For example, a study of consecutive exocytosis used temperature-sensitive mutants to accumulate secretory vesicles in the cell, and secretion was subsequently triggered by returning cells to the permissive temperature (80). In myoglobin, the rebinding of $\mathrm{CO}$ or $\mathrm{O}_{2}$ after bond breaking has been analyzed using flash photolysis (67) to generate synchronization. Stopped flow and $\mathrm{T}$ jump are other techniques that have been used for the synchronization of dynamic processes. Such synchronization attempts may allow a first step to be observed after triggering the system; however, two or more steps would be difficult to resolve owing to dephasing.

In contrast, the imaging of individual events allows unlimited consecutive steps to be observed in theory because no dephasing occurs. Thus, imaging at the molecular level not only removes the need for synchronization, it also allows for the imaging of consecutive, dependent interactions between molecular units.

Interactions between single molecules. One powerful example of the potential of studying the interactions of individual molecules comes from studies of the dynamics of the selection of tRNA on ribosomes (41, $81)$. In one study, researchers immobilized purified ribosomes on quartz slides via biotinylated mRNA, with ribosomes preformed with tRNA in the $\mathrm{P}$ or the A site. They used FRET between a single Cy3-tRNA in the P site and Cy5-tRNA in the A site to characterize the dynamic exchange between two distinct tRNA configurations on the ribosome, corresponding to the classical and hybrid states of the ribosome (41). They contrasted each singlemolecule recording with others by synchronizing to the first FRET signal above a minimum threshold (see Figure 3). This made it possible to compare different traces and create population histograms. At a single-molecule level, different states during tRNA selection and codon recognition were distinguished using inhibitors of different ribosomal states. The different states were achieved using nonhydrolyzable variants of GTP and ribosomes mutated within the GTPase activation site (the SRL), and by testing tRNA with mismatching bases (81). As a result, they found a new codon recognition state and evidence that the SRL is directly involved in GTP hydrolysis by EF-Tu. These interactions between tRNA and ribosomes are a striking example of dependent, consecutive events that can only be observed by imaging at the molecular level.

Molecular motors. The imaging of single events has contributed significantly to the study of two kinds of molecular motors: the motility motors that move cargo along the cytoskeleton and the metabolic motors that generate ATP from proton gradients. The initial studies of motility motors focused on the size of individual steps. This work has advanced to addressing mechanistic questions such as whether the motors move hand-overhand or like inchworms, or what the effect of tension is on the dynamics and energetics of the motors.

According to the hand-overhand model suggested for the walking mechanism of myosin and kinesin, the two heads of myosin/ kinesin walk by alternating which one is the leading head, similarly to how humans walk. One of the first tests of these models utilized DIC microscopy to image microtubules bound to immobilized kinesin molecules (82). The kinesin molecules were attached to glass coverslips by the distal end of the neck, so instead of twisting the stalk of kinesin, the rotation of the microtubule would be observed. The researchers never observed the microtubules performing the $180^{\circ}$ rotations that the hand-overhand model predicted. Therefore, they proposed an alternative, testable, inchworm model for the walking mode of kinesin. In the inchworm model, one kinesin head is always the leading one. The authors, 
a
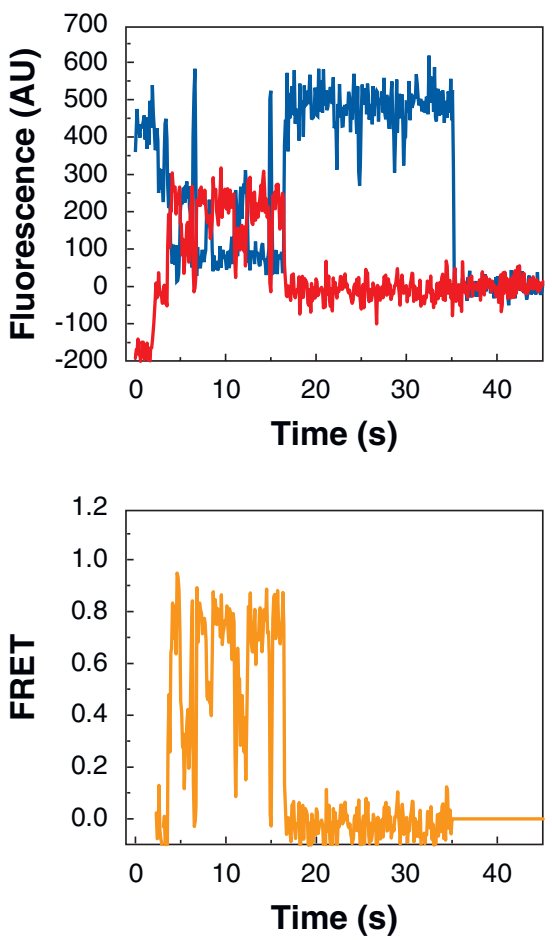

however, acknowledged that depending on the behavior of the neck-linker domain, an asymmetric hand-overhand model could not be ruled out (82).

Investigators further tested the models by using total internal reflection fluorescence microscopy and by labeling individual arms of myosin. It had been shown previously that the center of mass of myosin moves with 37-nm steps. The inchworm model predicted that the dye on the arm would move with 37-nm steps, whereas the hand-overhand model predicted alternating $(37+2 x)-\mathrm{nm}$ and $(37-2 x)-\mathrm{nm}$ steps, where $x$ is the dye's distance to the midpoint of myosin in the direction of the actin filament. The experimental observations supported the hand-overhand model (83). More recently, researchers demonstrated that as the motor moves, each head takes steps of $74 \mathrm{~nm}$, and the heads alternate taking the leading position, with each one being displaced from the other by $37 \mathrm{~nm}$ (84). Such observations are inconsistent with the inchworm model, b
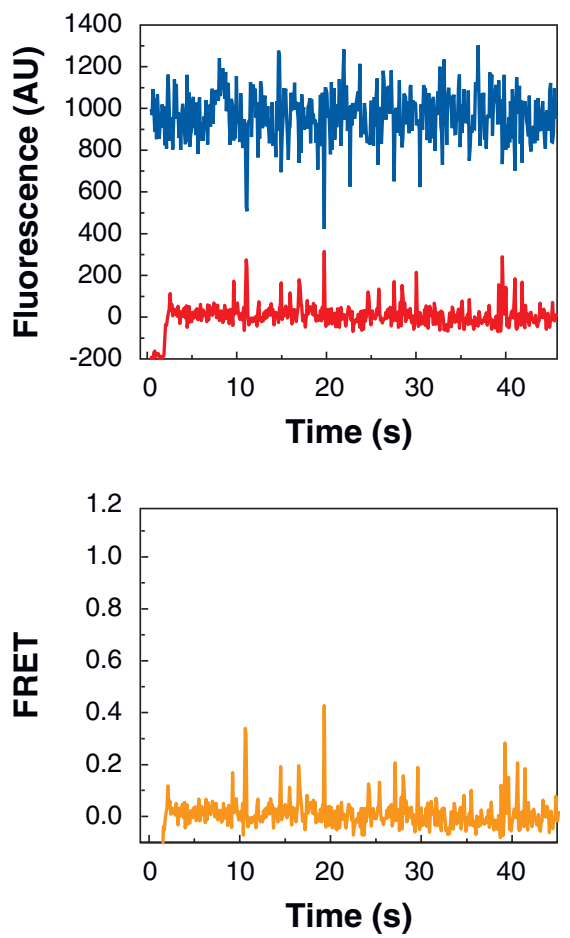

Figure 3

(a) (left panels)

Fluorescence resonance energy transfer (FRET) signal from binding and complete accommodation of cognate aa-tRNA on a ribosome.

Cy5-labeled tRNA

(EF-Tu-GTP-Phe-

tRNA $^{\text {Phe }}$ ) was

delivered to ribosomes initiated with Cy5-labeled fMet-tRNA ${ }^{\mathrm{fMet}}$ in the P site. (b) (right panels) When near-cognate aa-tRNAs (CUU instead of UUU) are delivered to a ribosome, tRNAs are rarely incorporated. Figure reprinted from Blanchard et al. (81).

which posits that one head maintains a leading position.

A similar analysis was applied to study the mechanism of action of kinesin. It had previously been established that the center of mass of kinesin moves with 8-nm steps (28). However, when researchers observed single molecules walking on microtubules $(83,85)$, the individual arms/heads take steps of $17 \mathrm{~nm}$ (85). Such steps are consistent with a handoverhand model for kinesin as well. Furthermore, a kinetic analysis of the dwell times between steps of single kinesin molecules indicated that the steps were either $17 \mathrm{~nm}$ or $0 \mathrm{~nm}$, also supporting the hand-overhand model.

Studies of the $\mathrm{F}_{0} \mathrm{~F}_{1}$-ATP synthase started addressing basic mechanistic questions, such as if it formed a rotary motor. The $\mathrm{F}_{0} \mathrm{~F}_{1}$-ATP synthase has been the subject of many single-molecule studies, the first published in 1997 in which the subcomplex $F_{1}$, carrying a fluorescent actin filament, was observed to 
a

Figure 4

Measurements of fluorescence resonance energy transfer (FRET) between subunits of the $\mathrm{F}_{0}-\mathrm{F}_{1}$-ATPsynthase revealed three different levels, each corresponding with a different rotation of the molecule.

(a) When driven by a proton gradient, the FRET cycles through levels 1,3 , and 2 , and $(b)$ when driven by ATP, the FRET cycles through levels 1, 2, and 3. Figure reprinted from Diez et al. (87).

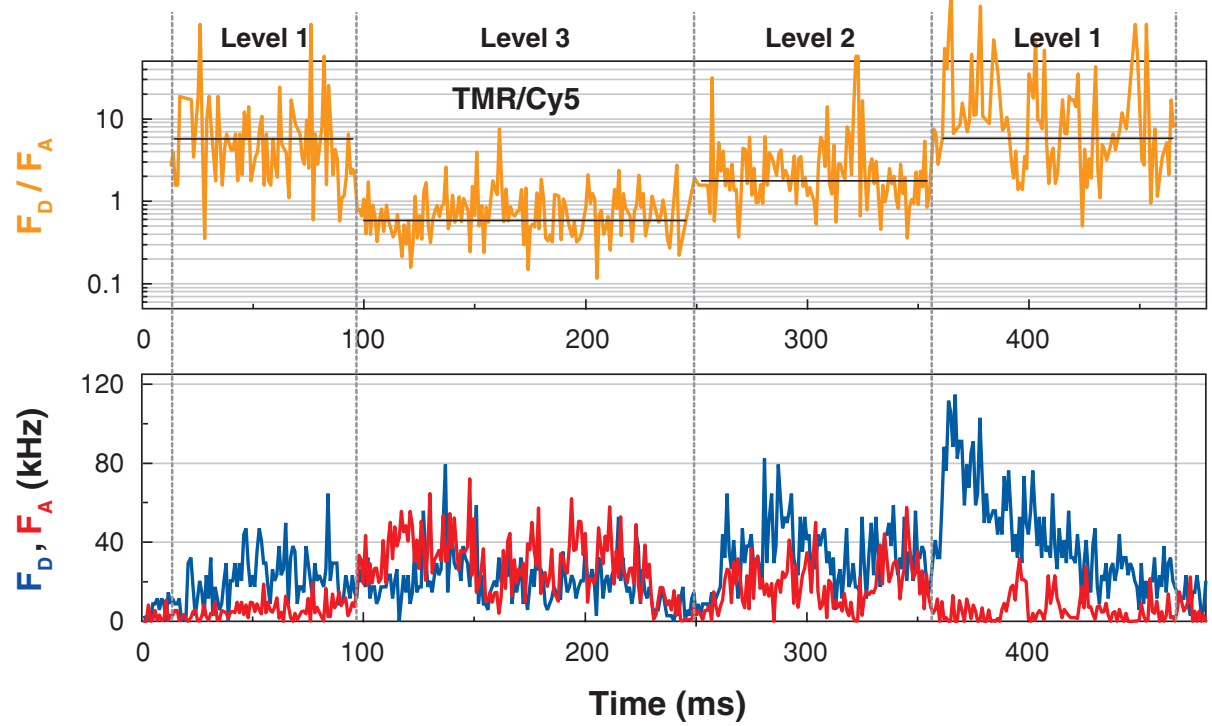

b

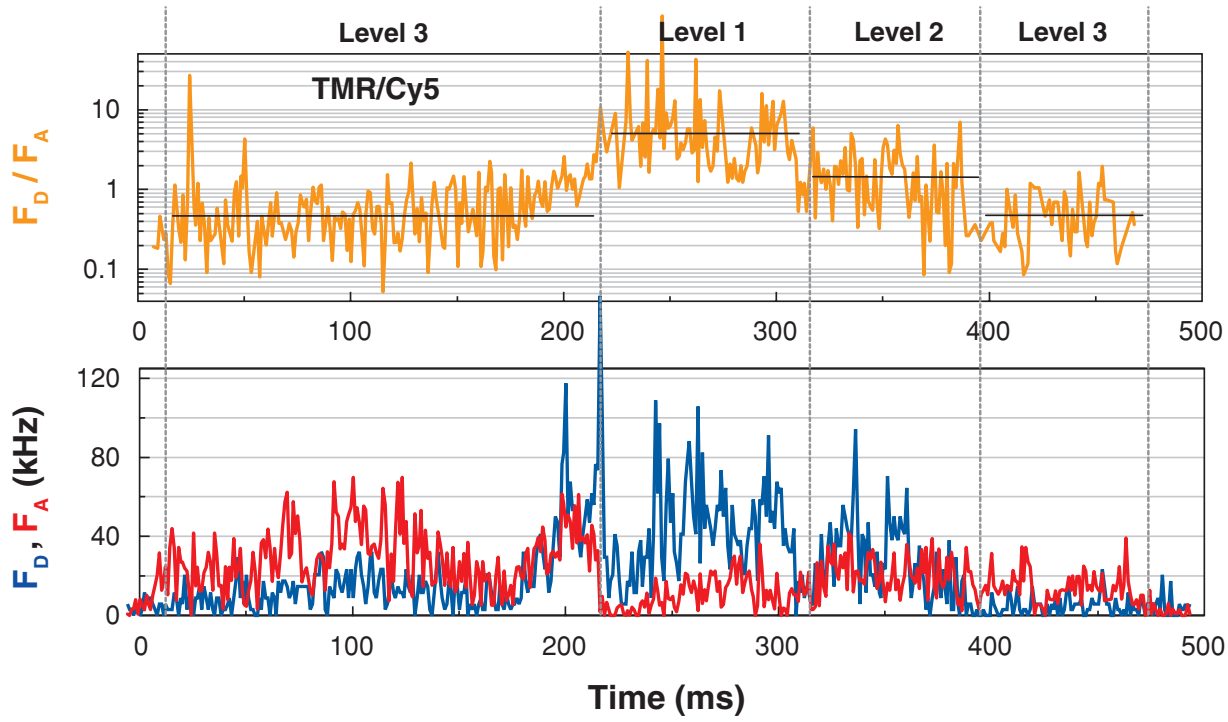

rotate during ATP hydrolysis (86). This paper was a milestone in the understanding of ATP synthase; the direct observation of the rotating $\mathrm{F}_{1}$-ATPase ended any further debate on whether ATP synthase worked by rotation.

Subsequent studies examined the energetics and dynamics of the substates. By labeling the subunits on $\mathrm{F}_{0}$ and $\mathrm{F}_{1}$ with TMR and
Rh110 and Cy5, respectively, three different levels of FRET could be studied. The $\mathrm{F}_{0} \mathrm{~F}_{1}$ ATP synthase moved through three step rotations of $120^{\circ}$, and each could be associated with a discrete level of FRET (see Figure 4). Investigators found that the temporal order by which the synthase went through each of the three FRET levels-the direction of 


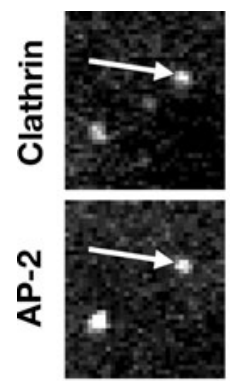

0

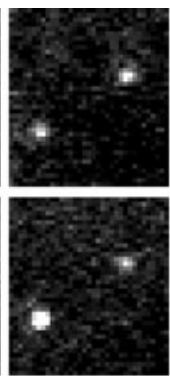

5

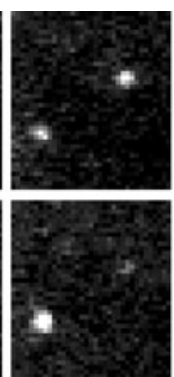

10

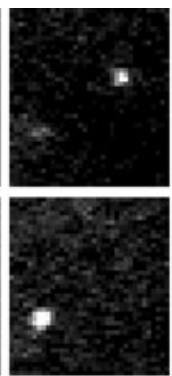

15

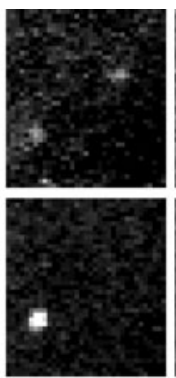

20

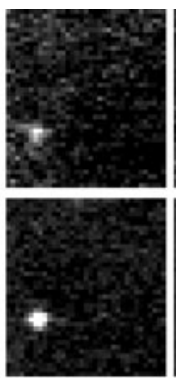

25

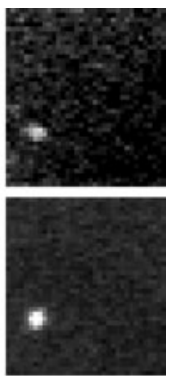

30

Time (s)

Figure 5

Endocytosis of clathrin and AP-2. When endocytosis was followed by total internal reflection fluorescence microscopy, the AP-2 always disappeared prior to the internalization of clathrin. Reprinted from Rappoport et al. (89) with permission.

rotation-was determined by whether the movement was predominantly driven by a proton gradient or by ATP hydrolysis (87). The ability to characterize the temporal order of the states of the $\mathrm{F}_{0} \mathrm{~F}_{1}$-ATP synthase depended on imaging at the single-molecule level.

Endocytosis. One of the pathways of endocytosis, the budding off of a vesicle from the membranes in cells, involves the protein clathrin. At the surface of the cell, clathrin is observed to localize with cargo molecules, as well as with a number effector molecules in endocytosis such as epsin (>60\% colocalization), dynamin, and adaptor protein complex AP-2 (>90\% colocalization). In some cell types the clathrin turns over approximately every $10 \mathrm{~min}$. Every minute, approximately $15 \%$ of the clathrin internalizes, which means that every second, approximately $0.25 \%$ internalizes, and when imaging at 10 frames per second, approximately $0.025 \%$ of the clathrin internalizes from frame to frame. Thus, at these rates of imaging, the bulk of clathrin at the surface is static, and the transient disappearance of clathrin is a relatively rare event. However, this rare transient state quantitatively accounts for the observed rates of endocytosis. An examination of the minority population of clathrin that is internalizing $(0.025 \%$ per frame) reveals that AP-2 cannot be detected in the vesicles $(88,89)$. In some circumstances the AP-2 dissociates from the clathrin prior to internalization (see Figure 5), and in some cases the clathrin septates off, leaving the AP-2 behind. If it was possible to tightly synchronize endocytosis, it might have been possible to detect this transient state from macroscopic measurements. However, by monitoring single events, it was not necessary to synchronize and the transient change was easily quantifiable.

\section{PITFALLS IN SINGLE-EVENT DETECTION}

There are a number of potential difficulties to studying single events, which we cover in detail below. First, the detection of single events usually pushes the limits of what can be detected. Larger fluorophores or multiple fluorophores are often used to increase the signal, leading to the following questions: How do we know if labeling is not interfering with the events, and how do we determine whether single or multiple molecules are being detected? Second, the first time single events are detected in any particular system usually involves a new assay. How can one determine if the assay is reporting the event that one is hoping to study? Third, if a single event 
Quantum dots

(QD): a

semiconductor nanocrystal, which is fluorescent is detected, how can one determine if it is a representative event rather than an aberrant anecdote?

\section{Are Labeled Molecules Functional?}

Most studies of single events involve attaching a tag to one of the molecules of interest. These tags have included silica beads, gold particles, and fluorophores. Each of these tags is limited by the number of photons it can generate. In general, the larger the tag, the greater the signal-to-background ratio and the greater the ease of detection. For example, an organic fluorophore may only be able to generate $10^{7}$ photons per second and photobleach after approximately $1 \mathrm{~s}$, whereas a larger fluorophore such as a quantum dot may generate $5 \times 10^{7}$ photons per second and may not photobleach during the time course of the experiment.

The restricted rate of photons limits the spatial resolution, the temporal resolution, and the ability to resolve one fluorophore from another. Thus, there are advantages to using larger tags that can generate more photons per unit time. However, larger tags are more likely to disrupt the movement of the molecule to which they are attached, and they are more likely to disrupt the interactions with other molecules of interest. The larger tags, such as quantum dots, are also usually multivalent, which raises concerns about crosslinking their targets (90).

How can one determine if the labeled protein is functional? A number of different criteria could be used, but not all of them are applicable to all circumstances. For in vitro measurements, the activity of labeled biomolecules can be compared with unlabeled ones. In the case of enzymes, the turnover rate can be contrasted for unaltered and tagged molecules $(41,87)$.

For measurements in cells, one test is whether the labeled molecules colocalize with the unlabeled molecules. A second test is to determine if the presence of the labeled molecules has any detectable adverse effects on the physiology of the processes to be stud- ied. For example, the studies of endocytosis described above required expressing various proteins (clathrin, epsin, adaptin) as fusions to fluorescent proteins. The expression of each of these had no detectable effect on the internalization of transferrin (Figure 6) (91). A third test is to see if the labeled protein can complement the loss of the endogenous protein (using small interfering RNA or a genetic mutation). Unfortunately, none of these tests is foolproof. A small interfering RNA might not reduce levels sufficiently to affect function. Alternatively, the labeled molecule may be functional but not introduced at proper levels to restore the macroscopic function. The development of methods for determining if a labeled molecule is functional is of extreme importance.

\section{Criteria for Identifying Single Molecules}

The experimental situation determines which approach can be used to test the detection of single molecules; for example, during live-cell imaging, the possibilities are different compared with when molecules are imaged in vitro. Therefore, we discuss the evidence that can be used in three different situations: immobilized molecules in vitro, freely diffusing molecules in vitro, and molecules in cells.

Several techniques exist for detecting whether two single molecules are bound. By labeling binding partners with dyes with different emission wavelengths, different fluorescence lifetimes, or different brightness, one can determine whether single detected molecules are bound or unbound (92-94). However, here we focus on how biomolecules labeled with dyes of a single type can be distinguished from two or more biomolecules bound to each other or aggregates of molecules all carrying dyes of the same type.

In vitro-immobilized molecules. Singlemolecule studies are often carried out on immobilized biomolecules, such as purified proteins or DNA. Immobilizing biomolecules 
a

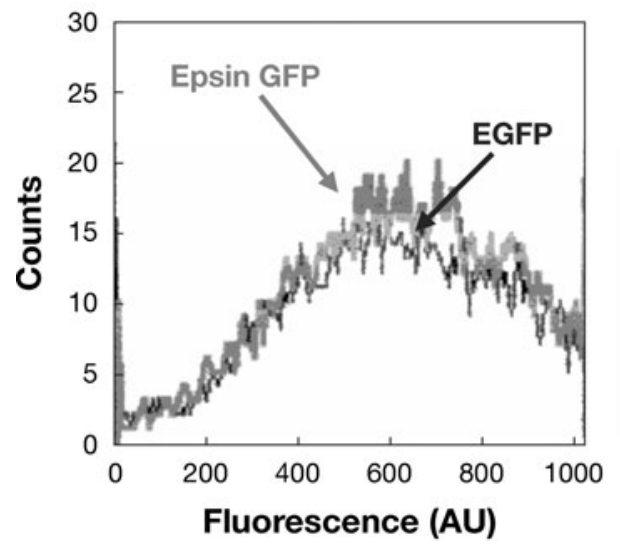

b

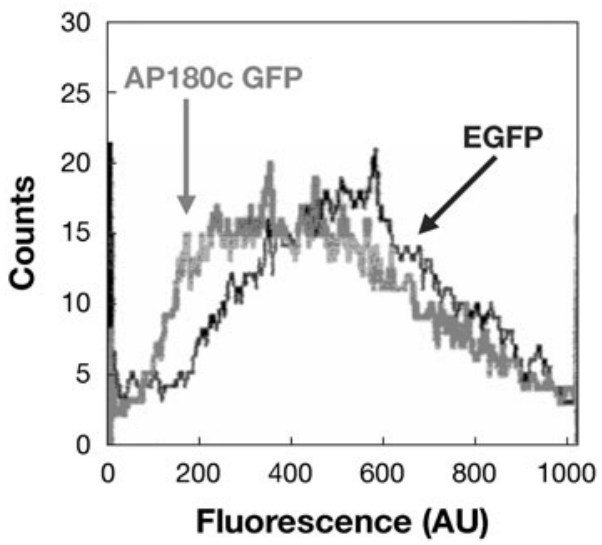

Figure 6

The endocytosis of labeled transferrin quantified using flow cytometry. A histogram is given for the amount of transferrin measured per cell. (a) The amount of transferrin endocytosed was not affected by the expression of epsin green fluorescent protein (GFP). (b) In contrast, expression of a dominant negative AP-180 (AP-180c) GFP reduced the endocytosis of transferrin. Abbreviation: EGFP, enhanced GFP. Figure adapted from Rappoport et al. (91) with permission.

makes imaging for extended times possible and also allows for induced changes to be applied, such as stopped flow (81) or photoactivation. For immobilized single molecules, one or several of the following criteria should be fulfilled.

Pbotobleaching. When molecules are illuminated until they photobleach, bleaching should occur in a single step. If the fluorescence spot originates from several molecules, a multistep decay or even an exponential decay is observed. When using FRET, if the donor bleaches, the fluorescence of both the donor and the acceptor ceases in a single step (95). However, if the acceptor bleaches, its fluorescence drops to background in a single step while the fluorescence of the donor simultaneously increases in a single step to its maximum, unperturbed, intensity level. This dual response is a specific indication of photobleaching of single donor/acceptor pairs. Sometimes, even when imaging a single molecule, one can observe a minor initial decay. This can be the result of the bleaching of molecules from out of the plane of focus. However, a single molecule should bleach down to background in a single step.

Fluorophores can enter long-lived dark states that can easily be mistaken for photobleaching. In fluorescent proteins, such dark states have been observed that can be switched on by irradiation at other wavelengths ( 96 , 97). It was recently reported that the fluorescent dye $\mathrm{Cy} 5$, which is often used as acceptor in single-molecule FRET measurements, can be switched back on from an apparent photobleached, dark state using 488, 514-, or 532-nm radiation. Moreover, a separate dark state was found, from which Cy5 quenches the fluorescence of the donor via energy transfer even stronger than from its fluorescent state (98). In addition to making FRET measurements more difficult to interpret, such dark states could create confusion as to whether single molecules are being observed.

Fluorescence intensity blinking. During illumination, fluorophores blink on and off. This behavior is routinely observed for organic and 


\section{Figure 7}

Blinking in the

fluorescent

emission of a single

quantum dot

between two

predominant

states. The

quantum dot was

illuminated with an

evanescent wave at

$442 \mathrm{~nm}(\mathrm{~S}$.

Wennmalm \&

S.M. Simon,

unpublished

observation).

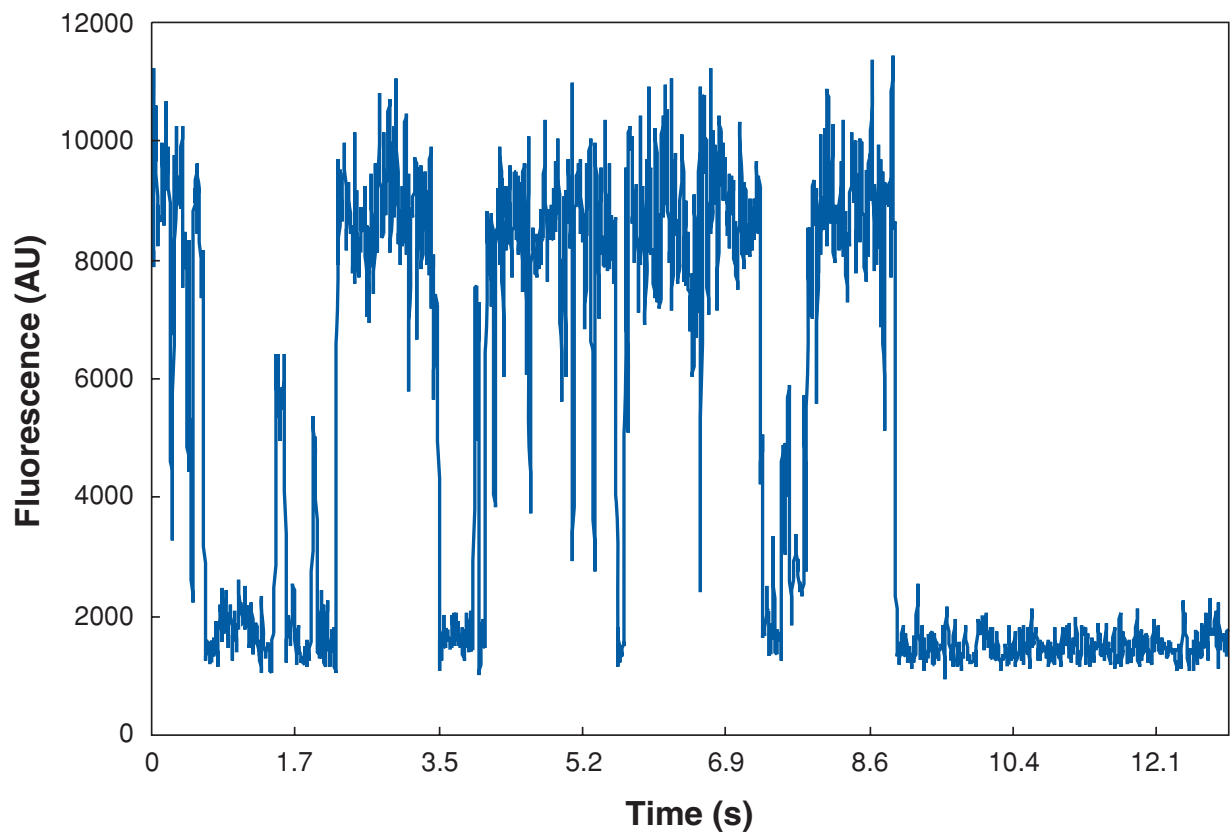

inorganic fluorophores and is fairly well described for the inorganic fluorescent semiconductors known as quantum dots $(99,100)$. The blinking sometimes limits the kinds of questions that can be addressed in single-molecule experiments, but it can also be used as a criterion for single-molecule detection. The detected fluorescent spots in a single-molecule experiment should blink between two distinct fluorescence levels (see Figure 7). Aggregates of two or a few molecules will still appear to blink, but two distinct levels will not be distinguished. Larger aggregates, or many fluorophores bound to the same biomolecule, will not appear to blink at all.

In some circumstances even single molecules can blink between more than two levels. In such cases, the blinking properties cannot be used to distinguish single molecules from multiple molecules. Moreover, multichromophoric systems exist, such as the protein $\beta$-phycoerythrin (101) or conjugated polymers $(102,103)$ that behave like single fluorophores in terms of blinking and photobleaching, even though they consist of many chromophores. They are, however, one single macromolecule and are not aggregates of molecules. Thus, despite the complex behavior in some fluorescent molecules, the fluorescence from a spot that is stably blinking between two distinct levels can only originate from a single molecule.

As in the case of bleaching, in a singlemolecule FRET experiment, if the donor enters the dark state, both donor and acceptor cease to fluoresce simultaneously. If the acceptor enters the dark state, the acceptor ceases to fluoresce while the donor simultaneously enters its maximum, free intensity level. Once again, the possibility of fluorophores entering long-lived dark states should be considered (98).

Fluorescence intensity histogram. In some experimental situations neither photobleaching nor blinking can be observed. This could occur if molecules can only be imaged for a limited time. In such a situation, evidence that single molecules are observed can be obtained by creating a fluorescence intensity histogram. First, one needs to generate an intensity histogram from a control sample in which the existence of single molecules can be independently tested. Then, if the histogram 
of the detected molecules has the same intensity distribution as the control, it is evidence that single molecules are detected. An intensity threshold can be defined, and individual fluorescence spots exceeding that threshold can be classified as being two or more molecules.

Antibunching. If molecules are imaged by the measurement of the fluorescence lifetime, antibunching is a signature of a single fluorophore. Antibunching is the decreased probability of detecting a second photon directly after (within fractions of the fluorescence lifetime of the dye) the detection of the first photon (104-106). The applicability of this approach is limited by the requirement of a relatively high intensity of excitation. Recently, researchers used antibunching to determine if an oligonucleotide was labeled with one or two Cy5 molecules (106).

Polarization. Another criterion for evaluating if the fluorescence is coming from a single immobilized chromophore is to measure the polarization of the emission. This approach takes advantage of the single dipole moment of individual molecules. If a single polarization direction from emitted light is observed, it is strong evidence of a single molecule. If the fluorescence at a particular spot is the product of multiple fluorophores, then as long as they are not exactly aligned, more than one emission polarization will be observed. Alternatively the total emission at certain polarization angles of excitation can be measured and will reveal whether a single fixed dipole is being detected (107).

In vitro freely diffusing molecules. In many studies, single molecules have been detected when diffusing freely in buffer solution through a (usually diffraction-limited) focused laser beam $(36-38,70)$. The high excitation intensity per area, combined with a low background from scattered photons owing to the use of a pinhole in the image plane, gives a large signal-to-background ratio. Moreover, because molecules usually are observed away from any glass surface, potential perturbing effects from surfaces are avoided $(108,109)$. On the downside, molecules only can be observed for as long as they reside in the detection volume. The duration can last anywhere from a few tens of microseconds to tens of milliseconds.

The short observation time also limits the possibility of determining whether single molecules, as opposed to aggregates of molecules, are being detected. Molecules can usually not be observed long enough to observe a single photobleaching step, and the criterion of blinking between two distinct levels cannot be applied because the fluorescence intensity of a molecule depends on its exact position in the Gaussian excitation profile.

Analogous to the situation with immobilized molecules, a fluorescence intensity histogram can be used to test whether single molecules are being detected. For freely diffusing molecules, this approach has been developed in detail and is known as fluorescence intensity distribution analysis (110) or photon counting histograms (111). The intensity distribution from single biomoleculeslabeled either with a single fluorophore or with a well-defined number of fluorophoresdiffers from an intensity distribution of biomolecules bound to each other or aggregates of molecules. Compared with a histogram of immobilized molecules, this approach gives broader distributions owing to the varying paths of molecules through the open volume (94). Fluorescence intensity distribution analysis has been combined with the analysis of polarization, emission wavelength, diffusion time, and fluorescence lifetime to increase sensitivity $(110,112,113)$. Clearly, if molecules are labeled with an unknown amount of fluorophores, it is more difficult to determine whether a detected burst originates from a single unit or an aggregate of units.

In vivo. Imaging of single molecules in live cells clearly has great possibilities, but it also 
poses great challenges. One challenge is how to introduce the molecules of interest to the cells. Molecules on the surface can be labeled directly. There are a few different approaches for labeling proteins in the cytosol. The molecules could be labeled noninvasively by letting cells express the proteins of interest fused to fluorescent proteins. Alternatively, the molecules of interest can be labeled in vitro and then invasively introduced into the cells. There are a variety of approaches, including microinjection, scrape loading, syringe loading, osmotic lysis of endosomes, and the use of cell-permeant peptides (114-117).

One critical problem for in vivo studies of single molecules is the endogenous fluorescence of cells. This raises the background fluorescence and thereby reduces the signal-tonoise ratio, which is almost always marginal even under optimal conditions. Thus, demonstrating that a signal in a cell originates from single molecules is not as easy as in in vitro systems.

Single fluorophore-labeled molecules. The possibility of using single-step bleaching and blinking as criteria for single-molecule detection in cells depends on the length of time a single molecule can be monitored. Sako et al. (56) imaged single-step bleaching events of fluorophores coupled to relatively immobile receptors on the cell surface (56). However, if molecules are imaged when diffusing relatively freely in the cytoplasm or in the nucleoplasm of cells $(57,66)$, even large proteins such as B-phycoerythrin only remain in focus for $\sim 240 \mathrm{~ms}$ (57). Photobleaching and blinking are observed on this timescale, but both are difficult to distinguish from a molecule that is leaving and re-entering the field of view. To test if a fluorescent puncta diffusing in three dimensions inside cells is a single molecule, one option is to create an intensity histogram and compare it with a control histogram. A second option is to measure the diffusion constant to see if the mass is consistent with that of a single molecule.
Multiple fluorophore-labeled molecules. The ability to image single molecules is often limited by the intensity of a single fluorophore. One way to increase the fluorescence intensity per molecule is to label the biomolecules with multiple fluorophores. This may be necessary in live-cell imaging to overcome endogenous background fluorescence, especially if fluorescent proteins are used, which are less bright than organic dyes. In an experiment applying this approach, the first indication of single-molecule detection is that a fluorescent spot has constant fluorescence intensity while it moves, and no other units associate to or dissociate from this spot.

With multiple dyes on a single biomolecule, neither blinking nor a single-step bleaching is observed. If each biomolecule has a defined number of fluorophores attached, one can use calibration of the fluorescence intensity from a single biomolecule to determine which detected spots originate from single or multiple biomolecules (118). In essence this is the same approach as the fluorescence intensity histogram comparison.

\section{DOES THE SIGNAL REPORTED BY OUR ASSAY REPRESENT THE SINGLE EVENT OF INTEREST?}

With the introduction of new techniquesparticularly techniques that push the existing limits of detection-it is the controls that give strength to the observations. For example, for the first recordings of single ion channels, researchers needed to demonstrate whether the fluctuations in the current were the consequence of a channel opening and closing, or if they reflected variations in the resistance of the seal between the glass and the membrane (119).

Evidence that an assay is reporting the event one is hoping to study can be given by showing that the system behaves as expected when exposed to different parameters. First, one can test whether a GTPdependent single-molecule system behaves as expected when exposed to nonhydrolyzable 
GTP analogs $(81,87)$. Second, when single molecules are detected, one can measure their emission spectrum and show that they are identical to the spectrum of the same type of molecules from a control experiment. Third, and perhaps the most important test, one can compare the distribution of the behavior observed from single events with the mean value obtained in a separate ensemble measurement. The following are some recent examples of observations of single events, focusing on the controls of the observations.

\section{Protein Translocation Across Membranes/Export}

Proteins that enter the secretory pathway of eukaryotic cells are made on ribosomes that are docked on the cytoplasmic side of the membrane of the endoplasmic reticulum. In 1989, researchers questioned whether nascent proteins enter the lumen of the endoplasmic reticulum by crossing through the hydrocarbon core of the lipid bilayer, or if they traverse the membrane through an aqueous channel? They tested two possibilities by taking membranes from the endoplasmic reticulum and fusing them to a planar lipid bilayer. The rationale was that if there are channels large enough to allow proteins to pass, they should be considerably larger than the channels that let ions pass, and therefore they should be amenable to study by the same kinds of techniques. With this approach, investigators observed a number of channels that were considerably larger than conventional ion channels (120). However, the critical test was to determine what was being observed: Were these channels involved in the movement of proteins across the membrane? The first test treated the membranes with puromycin, a tRNA analog that causes the release of nascent peptides from their biosynthetic ribosomes. The addition of puromycin resulted in the appearance of a channel in the membrane that was considerably larger than conventional ion channels. At low levels of puromycin $(200 \mathrm{nM})$, individual chan- nels could be observed, and at higher levels $(200 \mu \mathrm{M})$, there was a macroscopic increase in the conductance of the membrane (121). This could indicate the unplugging of polypeptides from channels. However, what if puromycin had some other effect on the membrane, causing the observed increase in conductance? To test this, researchers added puromycin to lipid bilayers that did not contain proteins from the endoplasmic reticulum, and they did not observe an effect. Furthermore, the addition of puromycin to the side of the membrane opposite the ribosomes (the equivalent of the lumen of the endoplasmic reticulum) had no effect on the conductance of the membrane; however, the subsequent addition of puromycin to the cytoplasmic side resulted in the release of the nascent polypeptides and the unplugging of the protein-conducting channels (121). Finally, the addition of the signal sequence, the amino acid segment of proteins that targets them for translocation, was by itself sufficient to open these channels (122).

Researchers are now studying these channels using structural biology (123-125), and they are studying the effect of signal sequences to gain insight into the physiological regulation of this pathway (126). Similar channels have been observed in the mitochondria $(127,128)$, the chloroplast (129), the bacterial plasma membrane (122), and even the outer bacterial membrane for the export of phage (130). Although the observation of a channel was sufficient to address the question of its existence, the critical test was in the controls to determine what was being observed.

\section{Membrane Exocytosis/Endocytosis}

The ability to observe the fusion of individual vesicles offers the potential of addressing questions such as when various proteins engage/disengage from the vesicles during docking and fusion, and if vesicles release all or none, or if they can partially release their contents. One of the first visualizations of a single vesicle was made for the release of a granule from the mast cell of the beige mouse 
(see Introduction, above, for a further discussion). These are mast cells from mice with a particular mutation that results in enormous micron-size granules.

Secretory vesicles, however, are usually one to two orders of magnitude smaller. Because these vesicles are smaller than the wavelength of light, it has proven problematic to follow individuals as they dock or fuse to the membrane. There is simply too much background fluorescence from other vesicles in the proximity of the membrane to detect single vesicles. One way to overcome this problem is to selectively excite a narrow plane of fluorophores $<100 \mathrm{~nm}$ above the cover slip using total internal reflection fluorescence to generate an evanescent wave (131-133). Narrowing the excitation limits the fluorescence to only those vesicles adjacent to the plasma membrane $(134,135)$.

The strength of the evanescent field decays exponentially from the membrane. Thus, when a vesicle with labeled fluorophores approaches the membrane, its fluorescence is observed to increase. After variable time, the lateral movement of the vesicle in the plane of the membrane ceases $(136,137)$, and the fluorescence is observed to rapidly expand laterally $(134,135)$.

One potential explanation for this lateral expansion of the fluorescence is exocytosis: the vesicle fused to the plasma membrane resulting in the rapid lateral diffusion of the vesicular contents. However, an alternative explanation is that as the vesicle gets closer to the membrane, and is excited more strongly by the evanescent field, photodamage results in lysis of the vesicle and thus lateral spread of the fluorophores.

How do we determine if the single event observed is reporting the event that we wish to study? Both explanations can account for the observations. One potential way to resolve the possible explanations is to selectively label only the membrane components of the vesicle (either lipids or proteins). Such a label would lead to two predictions that would distinguish between the models. First, if the vesicle was fusing to the plasma membrane, then it should deliver all its fluorophores to the plasma membrane. Thus, a measure of the total fluorescence of the vesicle (integrated over all of the pixels) would increase as the vesicle approaches and fuses, and then would remain constant because the fluorophores are now at a fixed distance from the membrane. Conversely, if the vesicle were lysed, then all of the fluorophores would not be resident in the plasma membrane. Second, if the vesicle were fusing to the plasma membrane, the fluorescent membrane components would then diffuse laterally in the plane of the plasma membrane. The spread of fluorescence should follow the relation of $D=\mathrm{cm}^{2} \mathrm{~s}^{-1}$. The area would increase linearly with time, and the slope of that increase would be the diffusion constant (138). If the vesicle had lysed, the membrane markers might remain in place or move away. However, they would not diffuse out laterally with the diffusion constant of a membrane protein in a lipid bilayer. Investigators have used such quantitative approaches to study the fusion of vesicles from the biosynthetic secretory pathway (139), the recycling pathway (140), and from lysosomes (141). The critical issue is that if the proper criteria can be established for determining if fusion is occurring, then the single events can be used to study the plethora of questions about exocytosis.

Endocytosis is the internalization of membrane components at the surface of a cell. Clathrin-mediated endocytosis occurs via 90-nm vesicles. What criteria can be used to determine if single endocytic events are being observed? When single components of the endocytic machinery, such as clathrin, are expressed as a fluorescent protein, they are observed in small puncta at the cell surface. Approximately $0.25 \%$ of these puncta disappear each second (142). Thus, this is a relatively rare event that could be explained a number of ways. First, it could be the result of photobleaching of the fluorophores. Second, it could be the result of labeled proteins dissociating from the membrane, rather than a 
puncta of proteins moving away together in an endocytic vesicle.

The following are three potential criteria that one can use to test if these events represent endocytosis. First, multiple endocytic markers can be labeled and followed at the same time. When one endocytic marker disappears, then all other markers at the same place disappear at the same time and the same rate. This would be consistent with them all leaving the evanescent field (143). Second, if the endocytic markers disappear from the evanescent field, but are still present with wide-field illumination, then they have not bleached and they have not dissociated, which is consistent with endocytosis (144). Third, if the rate of the disappearing events quantitatively accounts for the known rates of endocytosis, then this would be an additional criterion supporting endocytosis (143).

\section{WHEN IS A RARE ANECDOTAL EVENT SIGNIFICANT?}

One of the powerful aspects of studying single events is that one observes the entire spectrum of behaviors of individual events. Thus, rather than measuring the mean behavior of the system, a complete histogram of behaviors is generated. There are many examples in biology in which the single behavior is bimodal. For example, ion channels have discrete states of conductance. Information about the histogram of each of the conductance states under different conditions offers greater information than just the mean conductance. Upon examining individual molecules, it becomes clear that there are differences even between molecules that should otherwise be considered identical. One ion channel may be flickering rapidly between being open and close while another is in a period of relative quiescence. These differences may be the consequence of a number of different factors, for example, covalent posttranslational modifications such as phosphorylation or the binding of specific modulators such as ions or lipids.
However, this sensitivity to single molecules is also a potential drawback. If there are variations from individual to individual, then when an individual event/molecule is studied, a critical question is whether it is a representative event/molecule or an aberrant anecdote. This is not a trivial problem. Let us say one particular event is observed only $0.1 \%$ of the time. How should one determine if this particular event is of physiological significance or an aberration? Something that is present so rarely may be a single molecule that is damaged, modified, or targeted for degradation. The activity observed in a single event may be inconsequential to the physiology of the cell. There are numerous anecdotal examples that are inconsequential: They are simply the result of examining many single events. Dyson illustrates this point in the following: "In the course of any normal person's life, miracles happen at a rate of roughly one per month. During the time that we are awake and actively engaged in living our lives, roughly for eight hours each day, we see and hear things happening at a rate of about one per second. So the total number of events that happen to us is about thirty thousand per day, or about a million per month. With few exceptions, these events are not miracles because they are insignificant. The chance of a miracle is about one per million events. Therefore we should expect about one miracle to happen, on the average, every month" (145).

However, just because the observation of an event is rare does not mean that it is not significant. The activity of even a small minority population may be the significant population for understanding the physiological activity of this molecule. Many interactions in biology are transient, and as a result their activity is transient. The phosphorylation of key molecules in the nuclear envelope (such as the lamins) is a transient event: They are phosphorylated for a small fraction of the time. However, this transient phosphorylation is essential for the breakdown of the nuclear envelope during mitosis. 
An example of this quandary is the school bus problem. Let us say an alien force arrives on Earth and wants to determine the role of the rectangular yellow wheeled boxes observed on the North American continent. A series of aerial photos would predominantly capture school buses in large parking lots (Figure 8). A quantitative analysis would reveal that school buses strongly colocalize with barbed wire and guard dogs. Every so often a photograph would capture a single isolated school bus moving down the street picking up or dropping off kids. However, a quantitative analysis of the buses reveals that these represent only a few percent of the total school buses and therefore would be considered irrelevant to their normal physiological function (i.e., the once-a-month miracle).

In the above examples (the phosphorylation of lamins and the transport function of school buses), the significance of the transient events could be captured if the behavior of the lamins/school buses was synchronized (to the cell cycle or the school schedule). The advantage of detecting single events is that it is not necessary to synchronize the system or to know which events should be used for triggering the synchronization.

Conversely, when a particular event represents a substantial percentage of the population, it is still important to determine the significance of the behavior of the majority. This problem is particularly acute when a protein fused to a fluorophore is overexpressed. Our attention is drawn to the brightest signal. However, when expressed at high levels, the bulk of a protein may exceed the concentration of its normal binding partners. Thus, the localization or function of the protein of interest may be aberrant. An extreme example

Figure 8

Across the North American continent it is possible to observe large herds of wheeled yellow boxes.

These yellow boxes are found to overwhelmingly colocalize with barbed wire and guard dogs $(p<0.001)$ and thus are believed to play a role in maintaining security of the planet. of this is the accumulation of overexpressed proteins into inclusion bodies.

Even when a protein is not overexpressed, the behavior of its dominant population may not represent its dominant physiological function. For example, in a Xenopus oocyte, many of the nuclear pores are found in membrane sheets called annulate lamellae in the cytoplasm (146). These excess pores are stored up

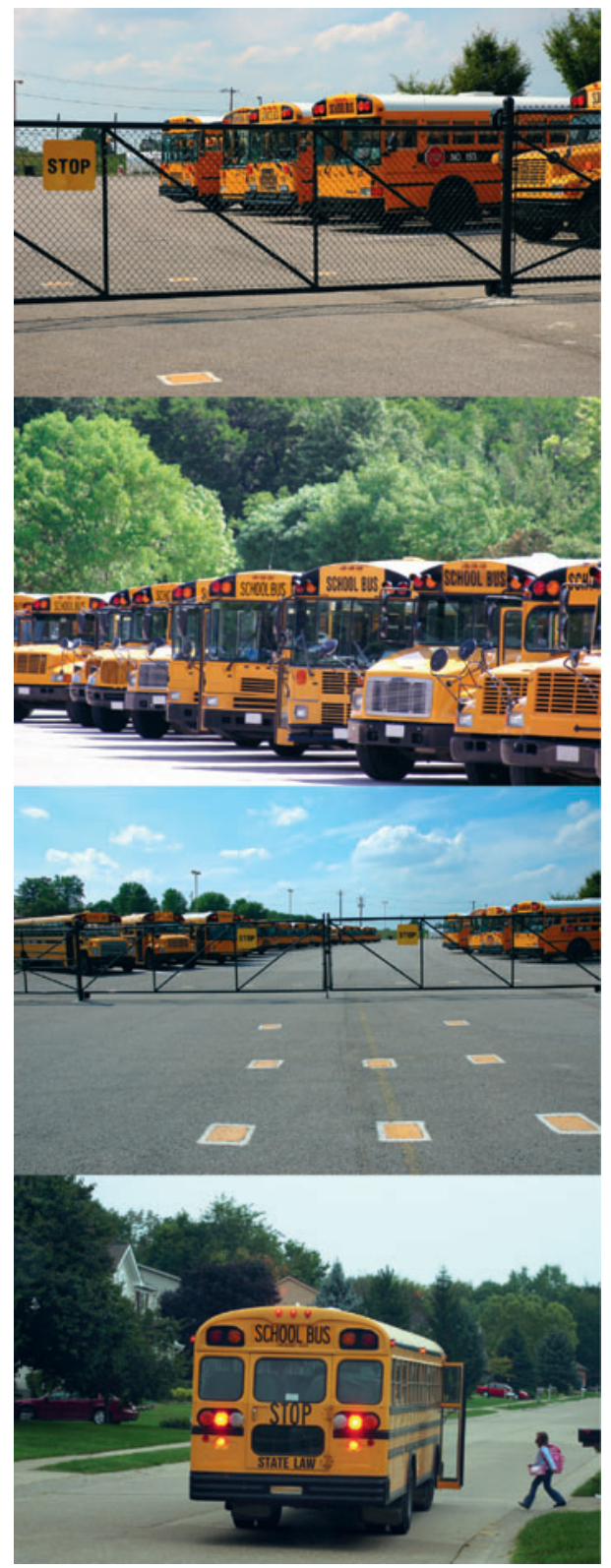


in preparation for when rapid nuclear division occurs after fertilization, and many pores in the nuclear envelope are required in a short time period. If one studies these pores in the cytoplasm, one would never get an indication that they were involved in nuclear transport.

\section{CONCLUSION AND OUTLOOK}

Researchers have gained much knowledge about cell mechanisms by using the direct imaging of single events at the molecular level. Using fluorescence imaging, they have analyzed interactions between biomolecules, between biomolecules and parts of the cell, dynamics within biomolecules, and processes such as endocytosis and exocytosis. Fluorescence imaging at the single-molecule level is the best technique today for directly observing the interaction and dynamics of biomolecules, and for revealing the mechanisms of molecular motors.
The majority of these studies have been performed in vitro, which so far best utilizes the spatial and temporal resolution of fluorescence imaging. An important step will be to facilitate the imaging of single molecular events inside living cells. For this several obstacles remain, such as labeling the molecules specifically, improving methods for determining if the labeled molecule is functional, controlling the concentration of the molecules of interest, and increasing the time period for which molecules can be kept in focus. For the latter problem, Gratton and coworkers (147) have suggested an approach that involves the automatic continuous adjustment of the focus of the objective. This technique currently allows tracking of particles over a range of 100 $\mu \mathrm{m}$ with 30 frames per second (147). When these remaining difficulties are overcome, it will be interesting to see what new discoveries can be performed from the fluorescence imaging of single events inside living cells.

\section{SUMMARY POINTS}

1. Investigators can use studies of single events to test models of biological mechanism.

2. Studies of single events facilitate the detection of infrequent subpopulations or transient events.

3. Studies of single events do not require macroscopic synchronization of a population to resolve the temporal order of events.

4. The strength of the signal can limit the studies of single events.

5. When studying a single event, it is important to resolve what is actually being reported by the assay. Specific criteria can determine when a single fluorophore is being detected.

6. Studies of single events must involve quantification to ensure that rare events are not insignificant anecdotal observations.

\section{FUTURE ISSUES}

Several improvements could greatly facilitate imaging of single events and biomolecules.

1. The development of small fluorophores, $\leq 3 \mathrm{~nm}$ diameter, with improved brightness, narrower emission spectra, and greater photostability. 
2. The development of more fluorophores that can be photoactivated or photoconverted as well as methods for coupling the photoactivation or photoconversion of fluorophores to activation of an enzyme.

3. The development of photostabilizers and triplet quenchers to facilitate the use of organic dyes.

4. The development of methods for quantitative microscopy. This includes methods for studying colocalization, for tracking molecules, and for quantifying population histograms rather than only mean values.

5. The development of cameras that will facilitate quantitative microscopy by being more linear as well as more sensitive without adding noise.

\section{ACKNOWLEDGMENTS}

We thank Jyoti Jaiswal and Phil Coffino for discussions. Work in our laboratory is supported by grants from the NSF (NSF BES 00520813) and NIH 1 P20 GM072015 to S.M.S. S.W. has a postdoctoral fellowship from the Knut and Alice Wallenberg Foundation.

\section{LITERATURE CITED}

1. Brown R. 1828. Edinb. New Philos. 7. 1828:358-71

2. Einstein A. 1905. Ann. Phys. 17:549-60

3. Magleby KL, Stevens CF. 1972. 7. Physiol. 223:173-97

4. Neher E, Sakmann B. 1976. Nature 260:799-802

5. Zimmerberg J, Cohen FS, Finkelstein A. 1980. Science 210:906-8

6. Zimmerberg J, Curran M, Cohen FS, Brodwick M. 1987. Proc. Natl. Acad. Sci. USA 84:1585-89

7. Breckenridge LJ, Almers W. 1987. Proc. Natl. Acad. Sci. USA 84:1945-49

8. Ringer S, Buxton DW. 1887. 7. Physiol. (Lond.) 8:15-19

9. Katz B, Miledi R. 1967. F. Physiol. (Lond.) 189:535-44

10. Llinás RR, Blinks JR, Nicholson C. 1972. Science 176:1127-29

11. Llinás RR, Steinberg IZ, Walton K. 1981. Biophys. F. 33:323-51

12. Simon SM, Llinás RR. 1985. Biophys. 7. 48:485-98

13. Weiss S. 1999. Science 283:1676-83

14. Moerner WE, Fromm DP. 2003. Rev. Sci. Instrum. 74:3597-619

15. Michalet X, Kapanidis AN, Laurence T, Pinaud F, Doose S, et al. 2003. Annu. Rev. Biophys. Biomol. Struct. 32:161-82

16. Allen RD, Metuzals J, Tasaki I, Brady ST, Gilbert SP. 1982. Science 218:1127-29

17. Schnapp BJ, Vale RD, Sheetz MP, Reese TS. 1985. Cell 40:455-62

18. Vale RD, Schnapp BJ, Mitchison T, Steuer E, Reese TS, Sheetz MP. 1985. Cell 43:623-32

19. Allen RD, Weiss DG, Hayden JH, Brown DT, Fujiwake H, Simpson M. 1985. 7. Cell Biol. 100:1736-52

20. Vale RD, Schnapp BJ, Reese TS, Sheetz MP. 1985. Cell 42:39-50

21. Kamiya N. 1981. Annu. Rev. Plant Physiol. Plant Mol. Biol. 32:205-36

22. Sheetz MP, Spudich JA. 1983. Nature 303:31-35

23. Yanagida T, Nakase M, Nishiyama K, Oosawa F. 1984. Nature 307:58-60 
24. Kron SJ, Spudich JA. 1986. Proc. Natl. Acad. Sci. USA 83:6272-76

25. Gelles J, Schnapp BJ, Sheetz MP. 1988. Nature 331:450-53

26. Howard J, Hudspeth AJ, Vale RD. 1989. Nature 342:154-58

27. Block SM, Goldstein LSB, Schnapp BJ. 1990. Nature 348:348-52

28. Svoboda K, Schmidt CF, Schnapp BJ, Block SM. 1993. Nature 365:721-27

29. Schnitzer MJ, Block SM. 1997. Nature 388:386-90

30. Hirschfeld T. 1976. Appl. Opt. 15:2965-66

31. Moerner WE, Kador L. 1989. Phys. Rev. Lett. 62:2535-38

32. Orrit M, Bernard J. 1990. Phys. Rev. Lett. 65:2716-19

33. Betzig E, Chichester RJ. 1993. Science 262:1422-25

34. Nguyen DC, Keller RA, Jett JH, Martin JC. 1987. Anal. Chem. 59:2158-61

35. Shera EB, Seitzinger NK, Davis LM, Keller RA, Soper SA. 1990. Chem. Phys. Lett. 174:553-57

36. Eigen M, Rigler R. 1994. Proc. Natl. Acad. Sci. USA 91:5740-47

37. Nie S, Chiu DT, Zare RN. 1994. Science 266:1018-21

38. Edman L, Mets U, Rigler R. 1996. Proc. Natl. Acad. Sci. USA 93:6710-15

39. Funatsu T, Harada Y, Tokunaga M, Saito K, Yanagida T. 1995. Nature 374:555-59

40. Yang H, Luo GB, Karnchanaphanurach P, Louie TM, Rech I, et al. 2003. Science 302:26266

41. Blanchard SC, Kim HD, Gonzalez RLJ, Puglisi JD, Chu S. 2004. Proc. Natl. Acad. Sci. USA 101:12893-98

42. Peters R, Peters J, Tews KH, Bahr W. 1974. Biochim. Biophys. Acta 367:282-94

43. Axelrod D, Koppel DE, Schlessinger J, Elson E, Webb WW. 1976. Biophys. F. 16:1055-69

44. Edidin M, Zagyansky Y, Lardner TJ. 1976. Science 191:466-68

45. Jacobson K, Derzko Z, Wu ES, Hou Y, Poste G. 1976. 7. Supramol. Struct. 5:565-76

46. Saffman PG, Delbruck M. 1975. Proc. Natl. Acad. Sci. USA 72:3111-13

47. De Brabander M, Nuydens R, Ishihara A, Holifield B, Jacobson K, Geerts H. 1991. 7 . Cell Biol. 112:111-24

48. Ghosh RN, Webb WW. 1994. Biophys. 7. 66:1301-18

49. Georgi A, Mottola-Hartshorn C, Warner A, Fields B, Chen LB. 1990. Proc. Natl. Acad. Sci. USA 87:6579-83

50. Suomalainen M, Nakano MY, Keller S, Boucke K, Stidwill RP, Greber UF. 1999. F. Cell Biol. 144:657-72

51. Schmidt T, Schutz GJ, Baumgartner W, Gruber HJ, Schindler H. 1995. 7. Phys. Chem. 99:17662-68

52. Schmidt T, Schutz GJ, Baumgartner W, Gruber HJ, Schindler H. 1996. Proc. Natl. Acad. Sci. USA 93:2926-29

53. Schwille P, Haupts U, Maiti S, Webb WW. 1999. Biophys. 7. 77:2251-65

54. Schwille P, Korlach J, Webb WW. 1999. Cytometry 36:176-82

55. Schutz GJ, Kada G, Pastushenko VP, Schindler H. 2000. EMBO 7. 19:892-901

56. Sako Y, Minoghchi S, Yanagida T. 2000. Nat. Cell Biol. 2:168-72

57. Goulian M, Simon SM. 2000. Biophys. 7. 79:2188-98

58. Seisenberger G, Ried MU, Endress T, Buning H, Hallek M, Brauchle C. 2001. Science 294:1929-32

59. Lakadamyali M, Rust MJ, Babcock HP, Zhuang X. 2003. Proc. Natl. Acad. Sci. USA 100:9280-85

60. McDonald D, Vodicka MA, Lucero G, Svitkina TM, Borisy GG, et al. 2002. F. Cell Biol. 159:441-52 
61. Yang W, Gelles J, Musser SM. 2004. Proc. Natl. Acad. Sci. USA 101:12887-92

62. Kubitscheck U, Grunwald D, Hoekstra A, Rohleder D, Kues T, et al. 2005. F. Cell Biol. $168: 233-43$

63. Babcock HP, Chen C, Zhuang X. 2004. Biophys. 7. 87:2749-58

64. Rothwell PJ, Berger S, Kensch O, Felekyan S, Antonik M, et al. 2003. Proc. Natl. Acad. Sci. USA 100:1655-60

65. Axelrod D, Ravdin P, Koppel DE, Schlessinger J, Webb WW, et al. 1976. Proc. Natl. Acad. Sci. USA 73:4594-98

66. Kues T, Dickmanns A, Luhrmann R, Peters R, Kubitscheck U. 2001. Proc. Natl. Acad. Sci. USA 98:12021-26

67. Austin RH, Beeson KW, Eisenstein L, Frauenfelder H, Gunsalus IC. 1975. Biochemistry 14:5355-73

68. Frauenfelder H, Sligar SG, Wolynes PG. 1991. Science 254:1598-603

69. Wennmalm S, Edman L, Rigler R. 1997. Proc. Natl. Acad. Sci. USA 94:10641-46

70. Eggeling C, Fries JR, Brand L, Gunther R, Seidel CAM. 1998. Proc. Natl. Acad. Sci. USA 95:1556-61

71. Jia Y, Sytnik A, Li L, Vladimirov S, Cooperman BS, Hochstrasser RM. 1997. Proc. Natl. Acad. Sci. USA 94:7932-36

72. Lu HP, Xun LY, Xie XS. 1998. Science 282:1877-82

73. van Oijen AM, Blainey PC, Crampton DJ, Richardson CC, Ellenberger T, Xie XS. 2003. Science 301:1235-38

74. Edman L, Foldes-Papp Z, Wennmalm S, Rigler R. 1999. Chem. Phys. 247:11-22

75. English BP, Min W, van Oijen AM, Lee KT, Luo G, et al. 2006. Nat. Chem. Biol. 2:87-94

76. Flomenbom O, Velonia K, Loos D, Masuo S, Cotlet M, et al. 2005. Proc. Natl. Acad. Sci. USA 102:2368-72

77. Velonia K, Flomenbom O, Loos D, Masuo S, Cotlet M, et al. 2005. Angew. Chem. Int. Ed. Engl. 44:560-64

78. Zhuang XW, Kim H, Pereira MJB, Babcock HP, Walter NG, Chu S. 2002. Science 296:1473-76

79. Davis PK, Ho A, Dowdy SF. 2001. BioTechniques 30:1322-31

80. Lew DJ, Simon SM. 1991. 7. Membr. Biol. 123:261-68

81. Blanchard SC, Gonzalez RL, Kim HD, Chu S, Puglisi JD. 2004. Nat. Struct. Mol. Biol. 11:1008-14

82. Hua W, Chung J, Gelles J. 2002. Science 295:844-48

83. Yildiz A, Forkey JN, McKinney SA, Ha T, Goldman YE, Selvin PR. 2003. Science 300:2061-65

84. Warshaw DM, Kennedy GG, Work SS, Krementsova EB, Beck S, Trybus KM. 2005. Biophys. 7. 88:L30-32

85. Yildiz A, Tomishige M, Vale RD, Selvin PR. 2004. Science 303:676-78

86. Noji H, Yasuda R, Yoshida M, Kinosita K. 1997. Nature 386:299-302

87. Diez M, Zimmermann B, Borsch M, Konig M, Schweinberger E, et al. 2004. Nat. Struct. Mol. Biol. 11:135-41

88. Rappoport JZ, Taha BW, Lemeer S, Benmerah A, Simon SM. 2003. F. Biol. Chem. 278:47357-60

89. Rappoport JZ, Benmerah A, Simon SM. 2005. Traffic 6:539-47

90. Jaiswal JK, Simon SM. 2004. Trends Cell Biol. 14:497-504

91. Rappoport JZ, Kemal S, Benmerah A, Simon SM. 2006. Am. 7. Physiol. Cell Physiol. 291:C1072-81 
92. Herten DP, Tinnefeld P, Sauer M. 2000. Appl. Phys. B 71:765-71

93. Widengren J, Kudryavtsev V, Antonik M, Berger S, Gerken M, Seidel CAM. 2006. Anal. Chem. 78:2039-50

94. Eggeling C, Berger S, Brand L, Fries JR, Schaffer J, et al. 2001. 7. Biotechnol. 86:163-80

95. Ha T, Enderle T, Ogletree DF, Chemla DS, Selvin PR, Weiss S. 1996. Proc. Natl. Acad. Sci. USA 93:6264-68

96. Dickson RM, Cubitt AB, Tsien RY, Moerner WE. 1997. Nature 388:355-58

97. Habuchi S, Ando R, Dedecker P, Verheijen W, Mizuno H, et al. 2005. Proc. Natl. Acad. Sci. USA 102:9511-16

98. Heilemann M, Margeat E, Kasper R, Sauer M, Tinnefeld P. 2005. 7. Am. Chem. Soc. 127:3801-6

99. Nirmal M, Dabbousi BO, Bawendi MG, Macklin JJ, Trautman JK, et al. 1996. Nature $383: 802-4$

100. Efros AL, Rosen M. 1997. Phys. Rev. Lett. 78:1110-13

101. Wu M, Goodwin PM, Ambrose WP, Keller RA. 1996. 7. Phys.Chem. 100:17406-9

102. VandenBout DA, Yip WT, Hu DH, Fu DK, Swager TM, Barbara PF. 1997. Science 277:1074-77

103. Barbara PF, Gesquiere AJ, Park SJ, Lee YJ. 2005. Acc. Chem. Res. 38:602-10

104. Basche T, Moerner WE, Orrit M, Talon H. 1992. Phys. Rev. Lett. 69:1516-19

105. Stoler D. 1974. Phys. Rev. Lett. 33:1397-400

106. Weston KD, Dyck M, Tinnefeld P, Muller C, Herten DP, Sauer M. 2002. Anal. Chem. 74:5342-49

107. Sosa H, Peterman EJ, Moerner WE, Goldstein LS. 2001. Nat. Struct. Biol. 8:540-44

108. Ha T, Enderle T, Chemla DS, Selvin PR, Weiss S. 1996. Phys. Rev. Lett. 77:3979-82

109. Osborne MA, Barnes CL, Balasubramanian S, Klenerman D. 2001. 7. Phys. Chem. B 105:3120-26

110. Kask P, Palo K, Ullmann D, Gall K. 1999. Proc. Natl. Acad. Sci. USA 96:13756-61

111. Chen Y, Muller JD, So PT, Gratton E. 1999. Biophys. 7. 77:553-67

112. Palo K, Mets U, Jager S, Kask P, Gall K. 2000. Biophys. 7. 79:2858-66

113. Palo K, Mets U, Loorits V, Kask P. 2006. Biophys. 7. 90:2179-91

114. McNeil PL, Murphy RF, Lanni F, Taylor DL. 1984. 7. Cell Biol. 98:1556-64

115. McNeil PL, Warder E. 1987. F. Cell Sci. 88(Pt. 5):669-78

116. McNeil PL. 1989. Methods Cell Biol. 29:153-73

117. Clarke MS, McNeil PL. 1992. 7. Cell Sci. 102:533-41

118. Shav-Tal Y, Singer RH, Darzacq X. 2004. Nat. Rev. Mol. Cell Biol. 5:855-61

119. Hamill OP, Marty A, Neher E, Sakmann B, Sigworth F. 1981. Pflügers Arch. 391:85-100

120. Simon SM, Blobel G, Zimmerberg J. 1989. Proc. Natl. Acad. Sci. USA 86:6176-80

121. Simon SM, Blobel G. 1991. Cell 65:371-80

122. Simon SM, Blobel G. 1992. Cell 69:677-84

123. Menetret J, Neuhof A, Morgan DG, Plath K, Radermacher M, et al. 2000. Mol. Cell 6:1219-32

124. Meyer TH, Ménétret JF, Breitling R, Miller KR, Akey CW, Rapoport TA. 1999. F. Mol. Biol. 285:1789-800

125. Beckmann R, Spahn CM, Eswar N, Helmers J, Penczek PA, et al. 2001. Cell 107:361-72

126. Wirth A, Jung M, Bies C, Frien M, Tyedmers J, et al. 2003. Mol. Cell 12:261-68

127. Henry JP, Chich JF, Goldschmidt D, Thieffry M. 1989. F. Membr. Biol. 112:139-47

128. Hill K, Model K, Ryan MT, Dietmeier K, Martin F, et al. 1998. Nature 395:516-21

129. Bulychev A, Pilon M, Dassen H, Van 't Hof R, Vredenberg W, De Kruijff B. 1994. FEBS Lett. 356:204-6 
130. Marciano DK, Russel M, Simon SM. 1999. Science 284:1516-19

131. Axelrod D. 1981. F. Cell Biol. 89:141-45

132. Thompson NL, Burghardt TP, Axelrod D. 1981. Biophys. 7. 33:435-54

133. Axelrod D, Burghardt TP, Thompson NL. 1984. Annu. Rev. Biophys. Bioeng. 13:247-68

134. Lang T, Wacker I, Steyer J, Kaether C, Wunderlich I, et al. 1997. Neuron 18:857-63

135. Steyer JA, Horstmann H, Almers W. 1997. Nature 388:474-78

136. Holz RW, Axelrod D. 2002. Ann. NY Acad. Sci. 971:232-43

137. Allersma MW, Bittner MA, Axelrod D, Holz RW. 2006. Mol. Biol. Cell 17:2424-38

138. Schmoranzer J, Goulian M, Axelrod D, Simon SM. 2000. 7. Cell Biol. 149:23-32

139. Schmoranzer J, Simon SM. 1999. Mol. Biol. Cell 10:A84

140. Lampson MA, Schmoranzer J, Zeigerer A, Simon SM, McGraw TE. 2001. Mol. Biol. Cell 12:3489-501

141. Jaiswal JK, Andrews NW, Simon SM. 2002. F. Cell Biol. 159:625-35

142. Rappoport JZ, Taha BW, Simon SM. 2003. Traffic 4:460-67

143. Rappoport J, Simon S, Benmerah A. 2004. Traffic 5:327-37

144. Merrifield CJ, Perrais D, Zenisek D. 2005. Cell 121:593-606

145. Dyson FJ. 2004. NY Rev. Books, Vol. 51:Issue 5, Mar. 25

146. Larabell CA, Chandler DE. 1988. Cell Tissue Res. 251:129-36

147. Ragan T, Huang HD, So P, Gratton E. 2006. F. Fluoresc. 16:325-36 


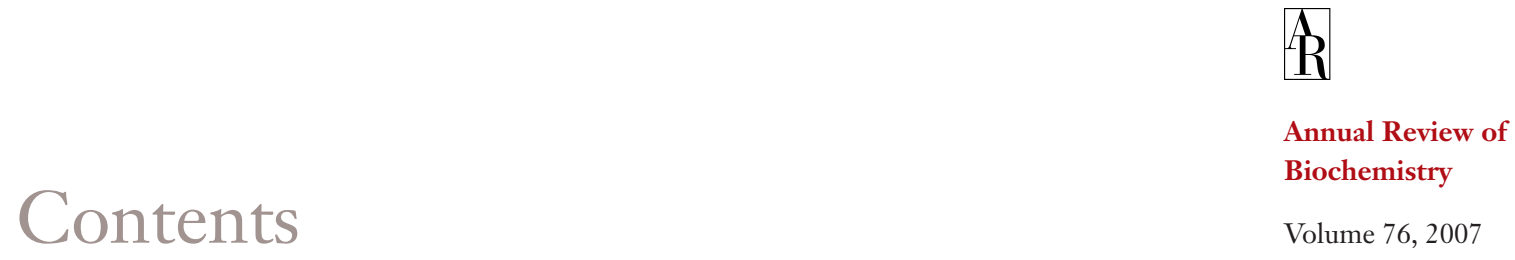

\section{Mitochondrial Theme}

The Magic Garden

Gottfried Schatz

DNA Replication and Transcription in Mammalian Mitochondria Maria Falkenberg, Nils-Göran Larsson, and Claes M. Gustafsson

Mitochondrial-Nuclear Communications Michael T. Ryan and Nicholas 7. Hoogenraad

Translocation of Proteins into Mitochondria Walter Neupert and Fohannes M. Herrmann

The Machines that Divide and Fuse Mitochondria Suzanne Hoppins, Laura Lackner, and Fodi Nunnari

Why Do We Still Have a Maternally Inherited Mitochondrial DNA?

Insights from Evolutionary Medicine

Douglas C. Wallace .781

Molecular Mechanisms of Antibody Somatic Hypermutation Javier M. Di Noia and Michael S. Neuberger 1

Structure and Mechanism of Helicases and Nucleic Acid Translocases

Martin R. Singleton, Mark S. Dillingham, and Dale B. Wigley

The Nonsense-Mediated Decay RNA Surveillance Pathway

Yao-Fu Chang, 7. Saadi Imam, Miles F. Wilkinson 51

Functions of Site-Specific Histone Acetylation and Deacetylation

Mona D. Shabbazian and Michael Grunstein 75

The tmRNA System for Translational Surveillance and Ribosome Rescue

Sean D. Moore and Robert T. Sauer 101

Membrane Protein Structure: Prediction versus Reality Arne Elofsson and Gunnar von Heijne 
Structure and Function of Toll Receptors and Their Ligands

Nicholas 7. Gay and Monique Gangloff

The Role of Mass Spectrometry in Structure Elucidation of Dynamic

Protein Complexes

Michal Sharon and Carol V. Robinson

Structure and Mechanism of the 6-Deoxyerythronolide B Synthase

Chaitan Khosla, Yinyan Tang, Alice Y. Chen, Nathan A. Schnarr,

and David E. Cane

The Biochemistry of Methane Oxidation

Amanda S. Hakemian and Amy C. Rosenzweig

Anthrax Toxin: Receptor Binding, Internalization, Pore Formation, and Translocation

Fobn A.T. Young and R. Fobn Collier

Synapses: Sites of Cell Recognition, Adhesion, and Functional

Specification

Soichiro Yamada and W. Fames Nelson

Lipid A Modification Systems in Gram-negative Bacteria

Christian R.H. Raetz, C. Michael Reynolds, M. Stephen Trent, and Russell E. Bishop

Chemical Evolution as a Tool for Molecular Discovery

S. Farrett Wrenn and Pebr B. Harbury

Molecular Mechanisms of Magnetosome Formation

Arash Komeili

Modulation of the Ryanodine Receptor and Intracellular Calcium Ran Zalk, Stephan E. Lebnart, and Andrew R. Marks

TRP Channels

Kartik Venkatachalam and Craig Montell

Studying Individual Events in Biology

Stefan Wennmalm and Sanford M. Simon

Signaling Pathways Downstream of Pattern-Recognition Receptors and Their Cross Talk

Myeong Sup Lee and Young-Foon Kim

Biochemistry and Physiology of Cyclic Nucleotide Phosphodiesterases:

Essential Components in Cyclic Nucleotide Signaling

Marco Conti and Joseph Beavo

The Eyes Absent Family of Phosphotyrosine Phosphatases: Properties and Roles in Developmental Regulation of Transcription fennifer femc and Ilaria Rebay 
Assembly Dynamics of the Bacterial MinCDE System and Spatial

Regulation of the Z Ring

Joe Lutkenhaus

Structures and Functions of Yeast Kinetochore Complexes

Stefan Westermann, David G. Drubin, and Georjana Barnes

Mechanism and Function of Formins in the Control of Actin Assembly

Bruce L. Goode and Michael F. Eck

Unsolved Mysteries in Membrane Traffic

Suzanne R. Pfeffer

Structural Biology of Nucleocytoplasmic Transport

Atlanta Cook, Fulvia Bono, Martin Finek, and Elena Conti

The Postsynaptic Architecture of Excitatory Synapses: A More

Quantitative View

Morgan Sheng and Casper C. Hoogenraad

\section{Indexes}

Cumulative Index of Contributing Authors, Volumes 72-76

Cumulative Index of Chapter Titles, Volumes 72-76

\section{Errata}

An online log of corrections to Annual Review of Biochemistry chapters (if any, 1997 to the present) may be found at http://biochem.annualreviews.org/errata.shtml 